\title{
A New Era of Diagnosis and Therapy in Acute Aortic Syndromes: The Mainz-Essen Experience (Part II)-Management and Outcomes
}

\author{
Eduardo Bossone, MD, $\mathrm{PhD}^{1, *(1)}$ Riccardo Gorla, MD, PhD ${ }^{2, *}$ Brigida Ranieri, $\mathrm{PhD}^{3}$
} Valentina Russo, MD ${ }^{4}$ Heinz Jakob, MD $^{5}$ Raimund Erbel, MD, MBA ${ }^{6}$

${ }^{1}$ Division of Cardiology, Antonio Cardarelli Hospital, Naples, Italy

2 Department of Cardiology, IRCCS Policlinico San Donato, San Donato Milanese, Milan, Italy

${ }^{3}$ Cardiovascular Imaging Division, IRCCS SDN (Scientific Institute for Research, Hospitalization and Healthcare), Naples, Italy

${ }^{4}$ Department of Advanced Biomedical Sciences, Federico II University of Naples, Naples, Italy

${ }^{5}$ Department of Thoracic and Cardiovascular Surgery, West German Heart Center, University of Duisburg-Essen, University Hospital

Essen, Essen, Germany

6 Institute for Medical Informatics, Biometry and Epidemiology (IMIBE), University of Duisburg-Essen, University Hospital Essen, Essen, Germany

Aorta (Stamford) 2021;9:201-214
Address for correspondence Eduardo Bossone, MD, PhD, Division of Cardiology, Antonio Cardarelli Hospital, Via Antonio Cardarelli, 980131 Naples, Italy (e-mail: eduardo.bossone@aocardarelli.it).

\author{
Abstract \\ Keywords \\ - acute aortic \\ syndromes \\ - Mainz-Essen \\ - acute aortic \\ dissection \\ - hybrid operating \\ room \\ - heart team concept
}

Over the years, the cardiovascular department of Johannes Gutenberg University in Mainz-West-German Heart Centre in Essen (Germany) designed and implemented the hybrid operating room (2003) along with advanced endovascular and surgical procedures, including the frozen elephant trunk technique. For the study purpose, the Mainz-Essen experience on acute aortic syndromes was summarized by considering original articles from single-center or multicenter studies performed at West German Heart Centre, Essen, Germany, or at the cardiovascular department of Johannes Gutenberg University, Mainz, Germany. We present the 35-year-long Mainz-Essen research, education, and patient management journey in creating an integrated multidisciplinary "Aortic Center" in the heart of Europe.

\section{Introduction}

Acute aortic syndromes (AAS) represent life-threatening medical emergencies needing a timely diagnosis and treatment (-Tables 1 and 2). ${ }^{1-5}$ Current European guidelines recommend urgent surgical repair for Type-A AAS, medical therapy alone for uncomplicated Type B AAS, and thoracic endovascular aortic repair (TEVAR) for complicated Type B AAS. ${ }^{1}$ Over the last decades, we have observed a substantial

\footnotetext{
* These authors contributed equally to this work.
}

received

June 5,2020

accepted after revision

July 2, 2021
DOI https://doi.org/

10.1055/s-0041-1739466.

ISSN 2325-4637. increase in surgical management for Type A acute aortic dissection (AAD; 79-90\%) along with a significant decline of surgical in-hospital mortality (25-18\%). On the other hand, no significant differences have been registered in Type B AAD overall in-hospital mortality $(12-14 \%, p=0.103)$. During this time, a larger number of complicated Type B AAD patients have been treated with endovascular interventions (7-31\%; - Supplementary Fig. S1). . $^{3,4}$

We discuss the evolution, achievements, and the development that the Mainz-Essen center has experienced since the inception of a dedicated hybrid theater, as well

\section{(c) 2021. The Author(s).}

This is an open access article published by Thieme under the terms of the Creative Commons Attribution License, permitting unrestricted use, distribution, and reproduction so long as the original work is properly cited. (https://creativecommons.org/licenses/by/4.0/)

Thieme Medical Publishers, Inc., 333 Seventh Avenue, 18th Floor, New York, NY 10001, USA 
Table 1 Acute aortic syndromes: definition and incidence ${ }^{1,2}$

\begin{tabular}{|c|c|c|c|}
\hline $\begin{array}{l}\text { Acute aortic } \\
\text { syndrome }\end{array}$ & Definition & Incidence $^{a}$ & Remarks \\
\hline AD (85-95\%) & $\begin{array}{l}\text { Disruption of the medial layer provoked by } \\
\text { intramural bleeding, resulting in separation } \\
\text { of the aortic wall layers and subsequent } \\
\text { formation of a TL and a FL with or without } \\
\text { communication. In most cases, an intimal } \\
\text { tear is the initiating condition }\end{array}$ & $2.6-6$ & $\begin{array}{l}\text { - The real incidence is difficult to define due } \\
\text { to pre-hospital mortality and/or missing } \\
\text { diagnosis } \\
\text { - The incidence is higher in men and increases } \\
\text { with age } \\
\text { - Types: } \\
\text { 67\% Type A } \\
\text { 33\% Type B }\end{array}$ \\
\hline IMH (10-25\%) & $\begin{array}{l}\text { Presence of hematoma in the media of the } \\
\text { aortic wall in the absence of a FL and intimal } \\
\text { tear }\end{array}$ & $\sim 1.2$ & $\begin{array}{l}\text { - Mainly in older patients } \\
\text { - Types: } \\
\text { 10-30\% Type A } \\
60-70 \% \text { Type B } \\
\text {-30-40\% of Type A IMH evolved into AD }\end{array}$ \\
\hline PAU (2-7\%) & $\begin{array}{l}\text { Ulceration of an aortic atherosclerotic plaque } \\
\text { penetrating through the internal elastic } \\
\text { lamina into the media }\end{array}$ & $\sim 2.1$ & $\begin{array}{l}\text { - Often multiple and different in size and } \\
\text { depth. } \\
\text { - More frequent in older age, male gender } \\
\text { and in patients with atherosclerotic } \\
\text { cardiovascular disease } \\
\text { - Types: } \\
\text { Rare in ascending aorta } \\
\sim 17.5 \% \text { aortic arch } \\
\sim 68 \% \text { descending aorta } \\
\sim 14 \% \text { thoracoabdominal transition }\end{array}$ \\
\hline
\end{tabular}

Abbreviations: $A D$, aortic dissection; $\mathrm{FL}$, false lumen; IMH, intramural hematoma; PAU, penetrating aortic ulcer; TL, true lumen. Note: Data from Erbel et $\mathrm{al}^{1}$ and Bossone et al. ${ }^{2}$

${ }^{\mathrm{a}}$ All data are per 100,000 person-years.

as a dedicated team to treat the aortic pathologies (-Tables $3^{6-26}$ and $4^{5,27-42}$; - Supplementary Table S1 and -Supplementary Fig. S2).

\section{Management and Outcomes of Acute Aortic Syndromes}

Irrespective of subsequent therapeutic interventions (open surgery and/or endovascular), patients presenting with AAD should promptly receive initial aggressive medical therapy to decrease heart rate $(<60 \mathrm{bpm})$, blood pressure (systolic blood pressure between 100 and $120 \mathrm{~mm} \mathrm{Hg}$ ), and $\mathrm{dP} / \mathrm{dT}$ (the instantaneous rate of left ventricular pressure rise during early systole and is a surrogate marker of left ventricular contractility). In this regard, intravenous $\beta$-blockers (propranolol, metoprolol, labetalol, or esmolol) are considered first-line agents; nondihydropyridine calcium channel blockers (verapamil and diltiazem) are potential alternatives in truly $\beta$-blocker intolerant individuals. Sodium nitroprusside (if needed) should be given in conjunction with $\beta$ blocker to lower blood pressure. Intravenous opiate analgesia should be implemented to control pain and related activation of the sympathetic nervous system. ${ }^{1}$

\section{Type A Acute Aortic Syndromes}

Immediate open surgical repair is recommended for Type $A$ AAD (mortality $\sim 22 \%)^{1,3,4}$ The in-hospital mortality rate among Type A AAD patients managed medically is prohibitively high ( $55 \%)$. Reported reasons for not performing surgery usually consist of advanced age, significant multiple comorbidities, malperfusion, extensive stroke, patient refusal, and death prior to planned surgery. ${ }^{1,3,4}$

\section{The Mainz-Essen Hybrid Operating Room Concept}

During aortic emergencies, the ability to intervene expeditiously is essential for an optimal outcome. In the 1980s, few patients with suspected AAS were transferred with an external computed tomography (CT) scan to Mainz University Hospital. Thus, patients had to be diagnosed in house, but time loss due to patient transfer to and from a remote catheterization suite to the surgical department caused a more than $10 \%$ casualty toll due to rupture or malperfusion aggravation. This led to the concept of transferring the AAS suspected patient right away into the operative suite with immediate transesophageal echocardiography (TEE) as the only preoperative diagnostic measure on the operating table.

Over the last two decades, the hybrid operating room (OR) revolutionized the approach to vascular emergencies. An individualized patient management through modern imaging techniques and advanced therapeutic approaches can be performed, permitting a timely intervention in a single clinical room. This elaborate concept was proposed at the University Hospital Essen in 1995, accepted and integrated into the construction plans for building a new heart center in 1997, which finally was realized within the first heart center in a German University Campus in Essen, opening in 2003.

The whole catheterization area was constructed as an operating theater including laminar air flow, enabling absolute sterile conditions for complete diagnostic, and 
Table 2 Classification systems of acute aortic syndromes ${ }^{1}$

\begin{tabular}{|c|c|c|}
\hline Classification & & Description \\
\hline \multirow[t]{2}{*}{ Stanford } & Type A & All dissections involving the ascending aorta irrespective of the site of tear \\
\hline & Type B & $\begin{array}{l}\text { All dissections that do not involve the ascending aorta; note that involvement of the } \\
\text { aortic arch without involvement of the ascending aorta in the Stanford classification } \\
\text { is labeled as Type B }\end{array}$ \\
\hline \multirow[t]{5}{*}{ DeBakey } & Category I & $\begin{array}{l}\text { Dissection tear in the ascending aorta propagating distally to include at least the } \\
\text { aortic arch and typically the descending aorta }\end{array}$ \\
\hline & Category II & Dissection tear only in the ascending aorta \\
\hline & Category III & Dissection tear in the descending aorta propagating most often distally \\
\hline & Category IIIa & Dissection tear only in the descending thoracic aorta \\
\hline & Category IIIb & Tear extending below the diaphragm \\
\hline \multirow[t]{5}{*}{ Svensson } & Class I & Classical dissection with true and false lumen \\
\hline & Class II & Intramural hematoma or hemorrhage \\
\hline & Class III & Subtle dissection without hematoma \\
\hline & Class IV & Penetrating atherosclerotic aortic ulcer \\
\hline & Class V & latrogenic or traumatic dissection \\
\hline \multirow{3}{*}{$\begin{array}{l}\text { Time course: from } \\
\text { symptom onset to } \\
\text { presentation (Erbel } \\
\text { et } \mathrm{al}^{1} \text { ) }\end{array}$} & Acute & $<14$ days \\
\hline & Subacute & 15-90 days \\
\hline & Chronic & $>90$ days \\
\hline
\end{tabular}

Note: Modified from Erbel et al. ${ }^{1}$

therapeutic options in cardiovascular emergencies. A 24/7 multidisciplinary "Aortic Team" (cardiac surgeons, cardiologists, and cardiac anesthesiologists) was initiated, which is the keystone feature to establish a timely diagnosis and to design the appropriate surgical procedure (-Fig. 1). ${ }^{26}$

The catheterization table integrates all the elements of a surgical table, providing the possibility of performing surgery on the same table without moving the patient. ${ }^{26,43}$

Nowadays, the European Society of Cardiology (ESC) guidelines consider the hybrid OR as the optimal environment for implementing new therapeutic options. ${ }^{1}$ The Aortic Team, with the help of a multidisciplinary approach, modern imaging systems, careful planning, and professional expertise was able to develop new therapeutic approaches and modern surgical procedures. Jakob and Tsagakis ${ }^{44}$ were the leading pioneers of the frozen elephant trunk (FET) technique, a modern surgery combining replacement of the ascending aorta, repair of the aortic arch, and endografting of the proximal descending aorta ${ }^{7,15,44}$ (- Supplementary Fig. $\mathbf{3 3})^{6}$

It could be demonstrated that in-hospital mortality did not become worse by this more complex surgical approach; rather long-term outcomes improved in terms of fewer reinterventions/reoperations downstream. ${ }^{9,14,23}$ Moreover, proof could be generated that positive remodeling and false lumen thrombosis take place in more than $95 \%$ of acute, and in more than $90 \%$ of chronic Type I aortic dissection cases, in the region around the descending aorta stent-graft. ${ }^{10,12}$ The international E-vita registry, inaugurated by the Essen group, with the participation of 20 European cardiothoracic centers, confirmed the results from Essen. Indication for the use of
FET was able to be extended, leading, based on observed results, to modifications in surgical strategies throughout all participating centers. ${ }^{7-9,11,17,45,46}$ Based on these prerequisites, additional aspects of surgical treatment options and safety tools for implantation of FET could be elaborated in Essen, including the very fast and safe cannulation technique in threatening emergency situations, and the nowadays angioscopic evaluation of the thoracoabdominal aorta during hypothermic circulatory arrest, to control the FET landing zone deep down in the surgically invisible mid-descending thoracic aorta. ${ }^{24,47}$ With this advanced knowledge about the pathophysiology and pathomorphology of acute Type I or II aortic dissection, it became possible to differentiate the indication for FET or lack of indication. When the dissection process stops within the ascending aorta (the classic DeBakey Type II), or within the arch (the Essen modified Type II classification), hemiarch and total arch replacement is sufficient without FET, resulting in stable long-term results without the need for late reintervention. ${ }^{25}$ Thus, for patients with AAS, a significant improvement in the management of acute aortic emergencies was achieved, including aortic dissection, leading to the so-called "Essen hybrid" algorithm (-Fig. 2). ${ }^{26}$

All patients with suspected or proven aortic dissection are transported directly into the hybrid OR. Furthermore, with the hybrid OR, it is possible to investigate a potential malperfusion site without time loss. The coronary, cerebral, visceral, and peripheral vascular branches are investigated through angiography in stable patients, while the anesthesiologist is instrumenting the patient and controlling blood pressure, the scrub nurse is preparing the instrument tables, 


\begin{tabular}{|c|c|c|c|c|c|c|c|c|}
\hline 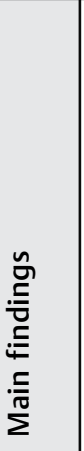 & 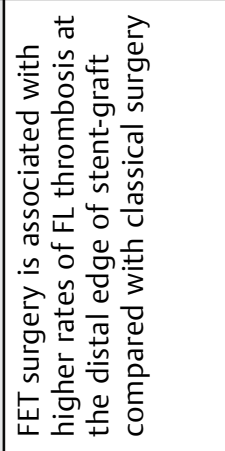 & 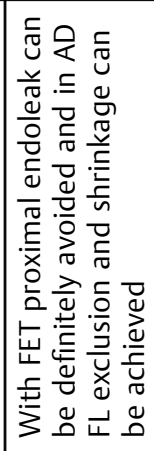 & 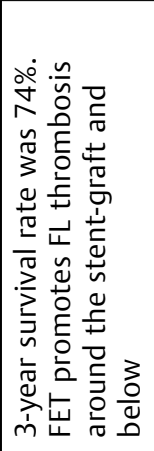 & 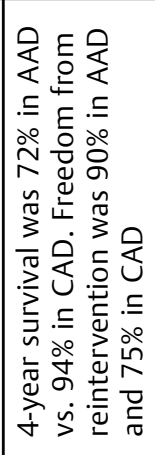 & 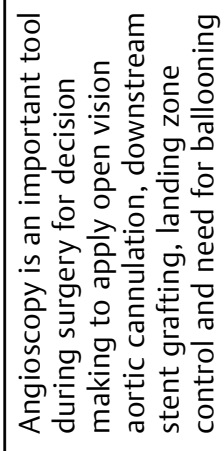 & 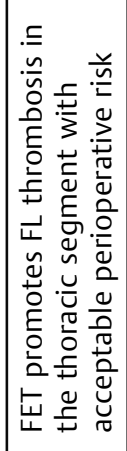 & 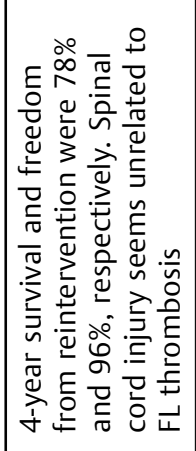 & 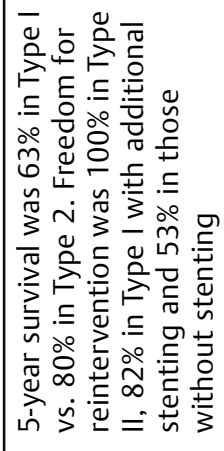 \\
\hline 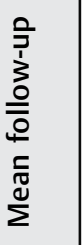 & 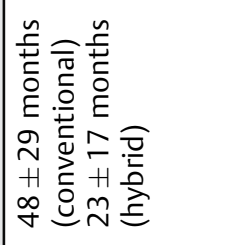 & 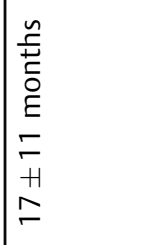 & 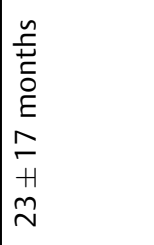 & 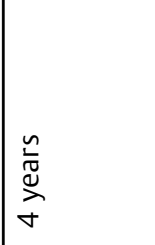 & $\S$ & 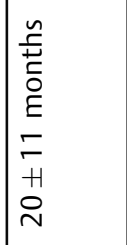 & 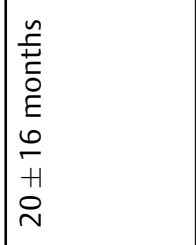 & 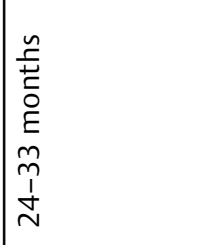 \\
\hline$\stackrel{\frac{.}{0}}{\circ}$ & 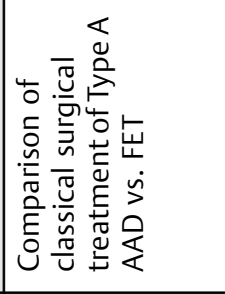 & 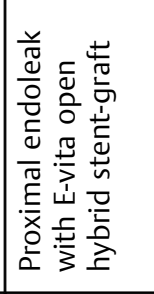 & 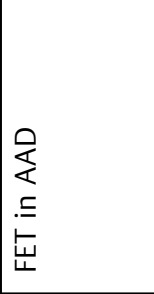 & 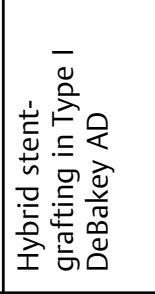 & 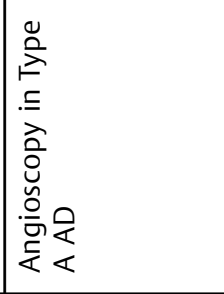 & 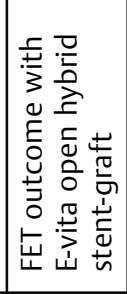 & 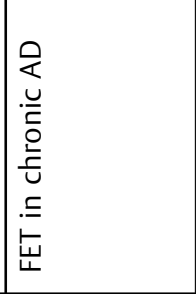 & 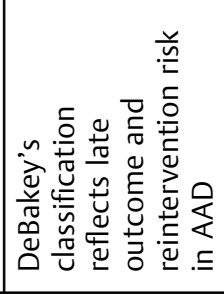 \\
\hline 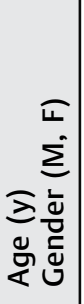 & 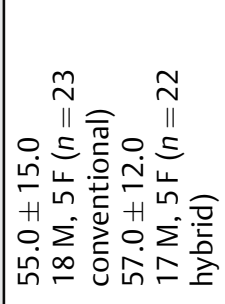 & 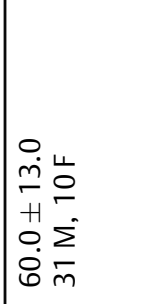 & 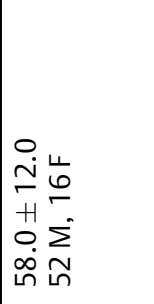 & & 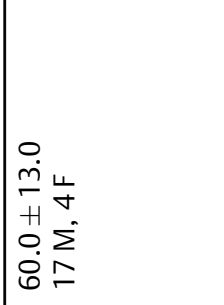 & 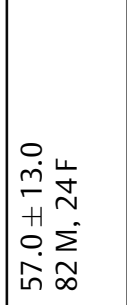 & 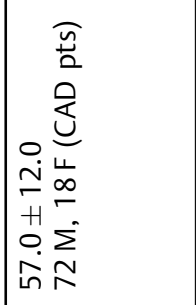 & 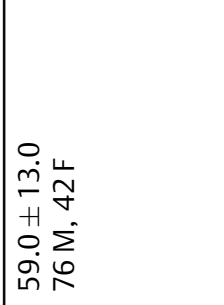 \\
\hline 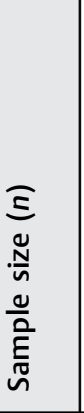 & 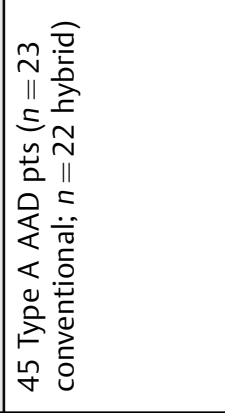 & 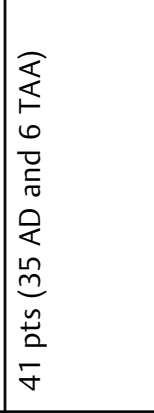 & 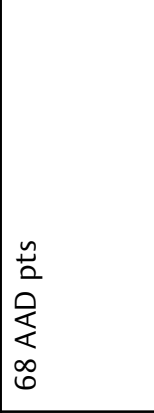 & 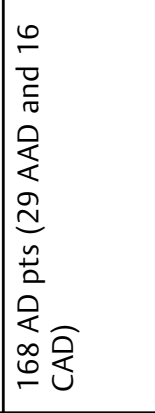 & 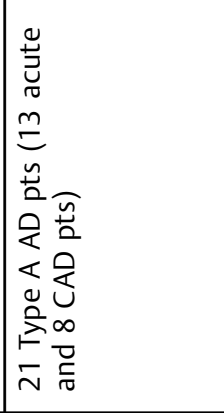 & 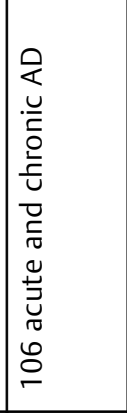 & 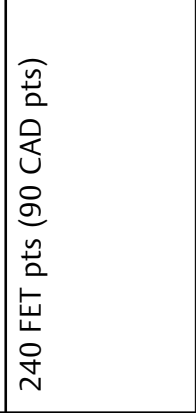 & 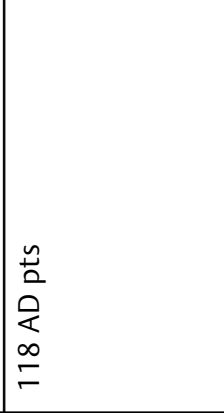 \\
\hline 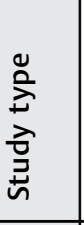 & 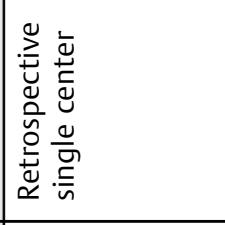 & 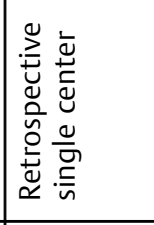 & 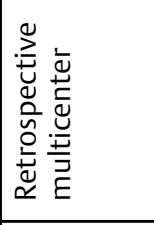 & 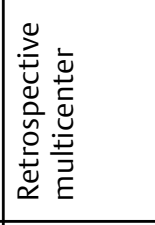 & 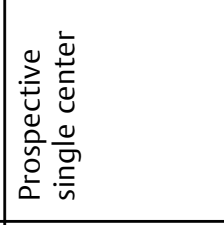 & 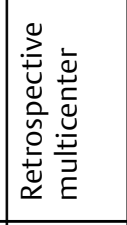 & 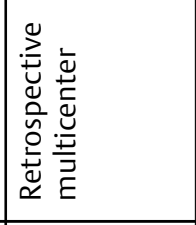 & 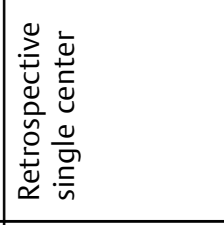 \\
\hline 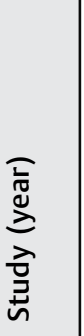 & 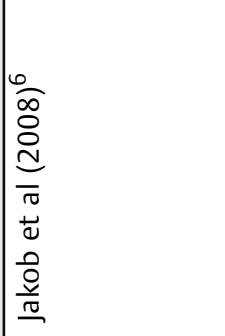 & 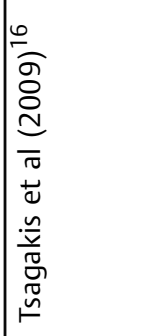 & 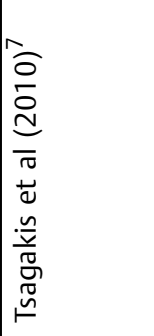 & 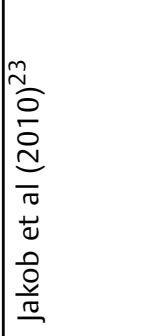 & 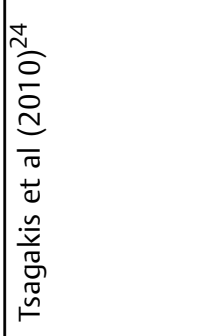 & 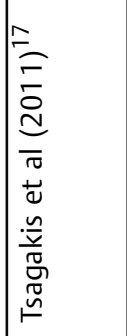 & 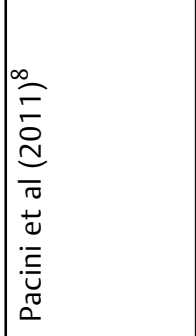 & 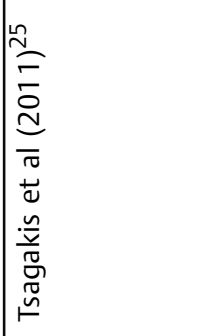 \\
\hline
\end{tabular}




\begin{tabular}{|c|c|c|c|c|c|c|c|c|}
\hline 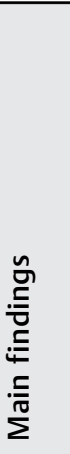 & 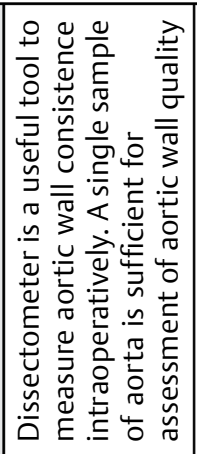 & 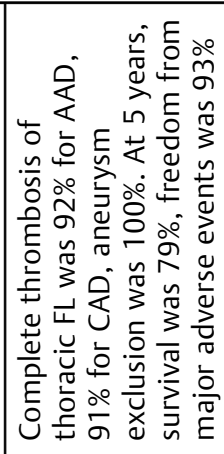 & 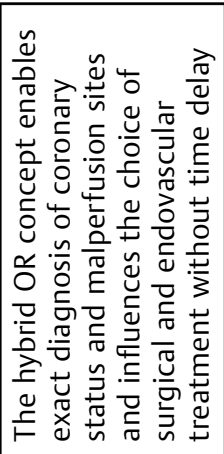 & 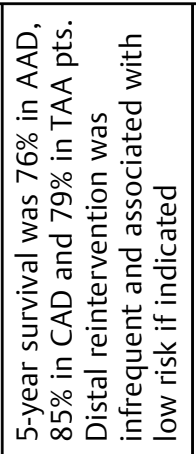 & 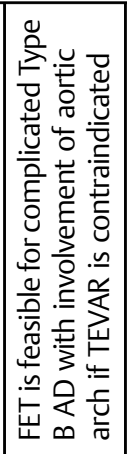 & 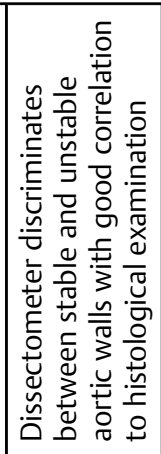 & 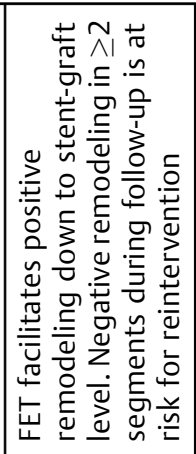 & 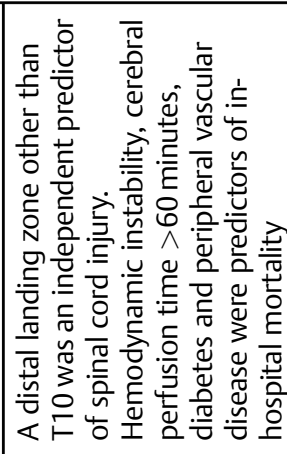 \\
\hline $\begin{array}{l}\frac{0}{3} \\
\sum_{0}^{1} \\
\frac{0}{0} \\
\substack{c \\
\frac{\pi}{\pi} \\
\sum}\end{array}$ & 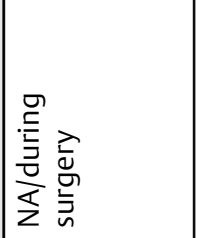 & 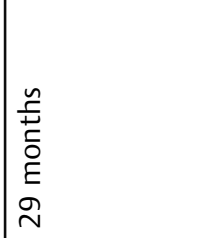 & $\Sigma$ & $\Sigma$ & 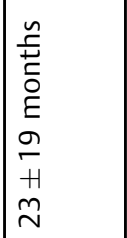 & $\Sigma$ & 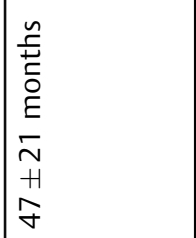 & 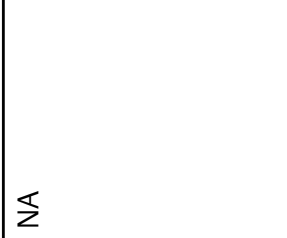 \\
\hline$\stackrel{\frac{0}{0}}{\circ}$ & 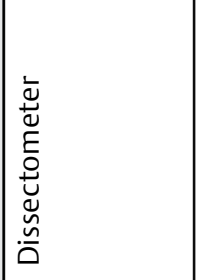 & 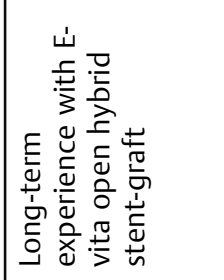 & 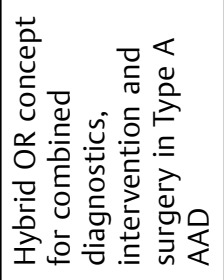 & 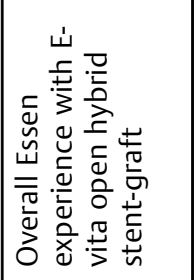 & 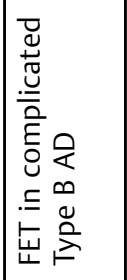 & 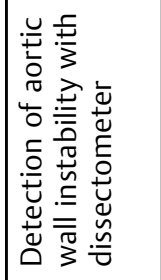 & 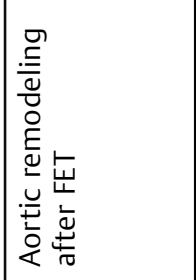 & 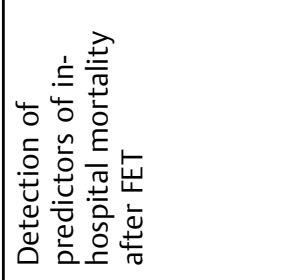 \\
\hline 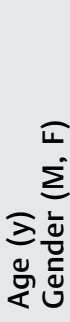 & 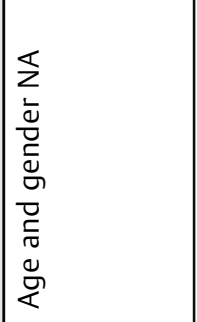 & 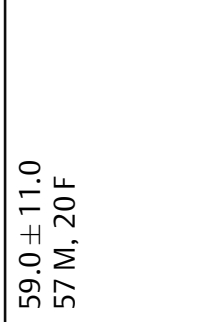 & 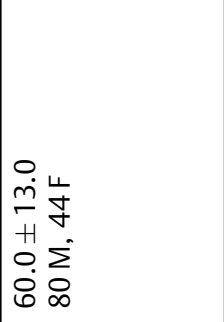 & 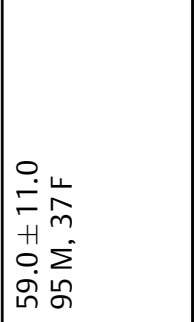 & 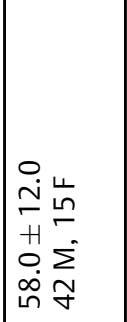 & 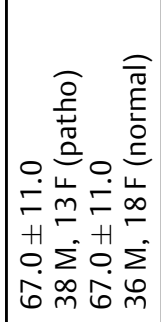 & 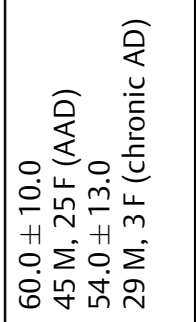 & 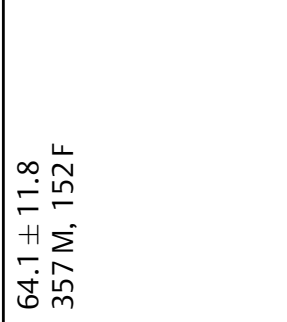 \\
\hline $\begin{array}{l}\text { ఏ } \\
\stackrel{N}{n} \\
\frac{0}{0} \\
\frac{0}{n} \\
\text { ñ }\end{array}$ & 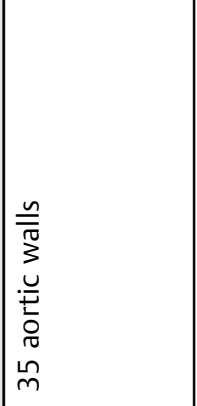 & 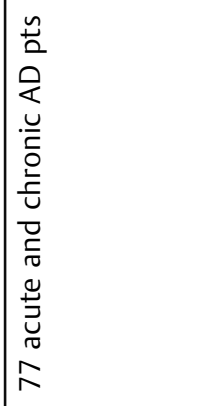 & 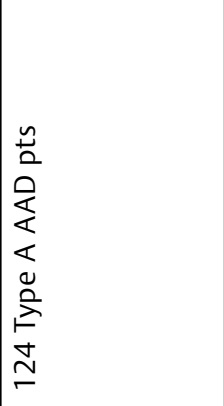 & 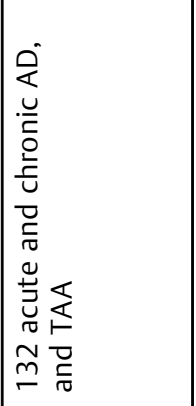 & 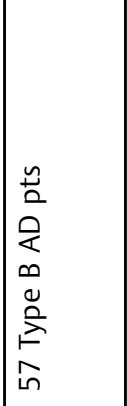 & 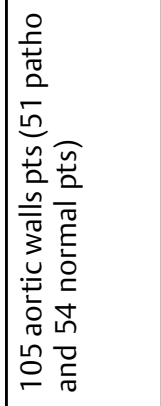 & 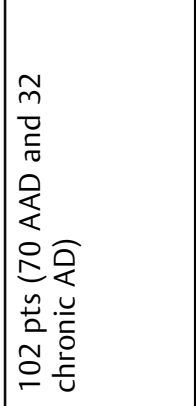 & 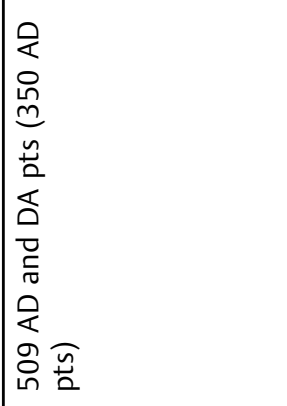 \\
\hline 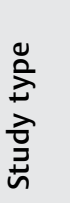 & 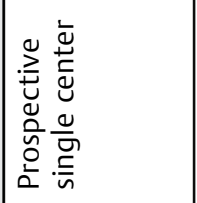 & 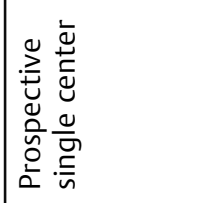 & 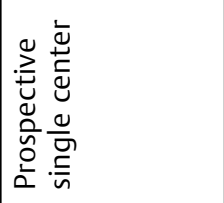 & 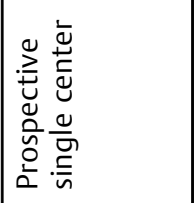 & 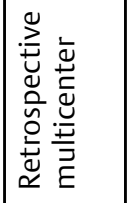 & 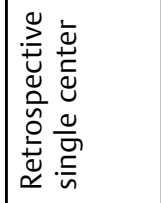 & 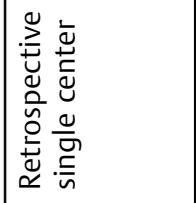 & 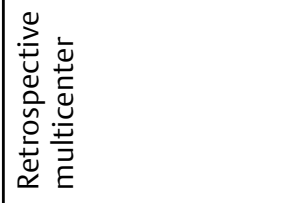 \\
\hline 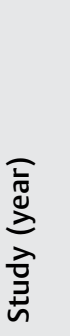 & 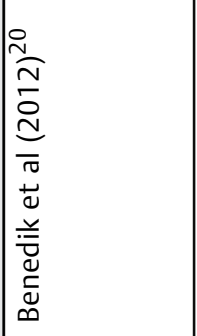 & 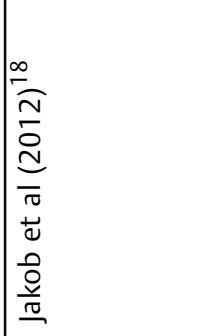 & 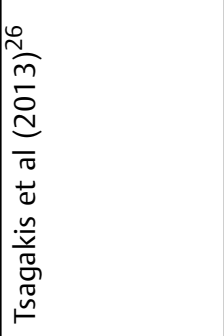 & 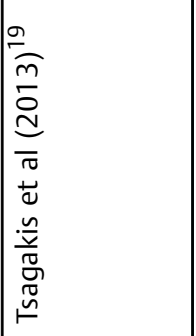 & 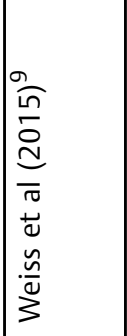 & 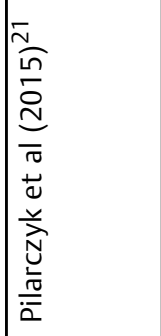 & 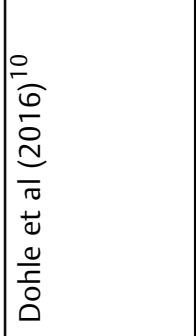 & 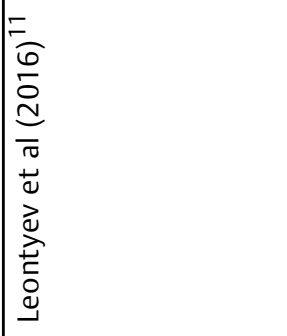 \\
\hline
\end{tabular}




\begin{tabular}{|c|c|c|c|c|c|}
\hline 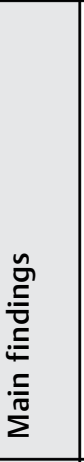 & 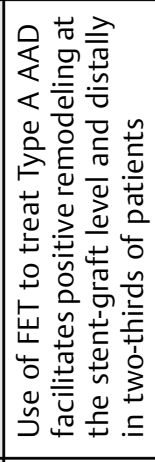 & 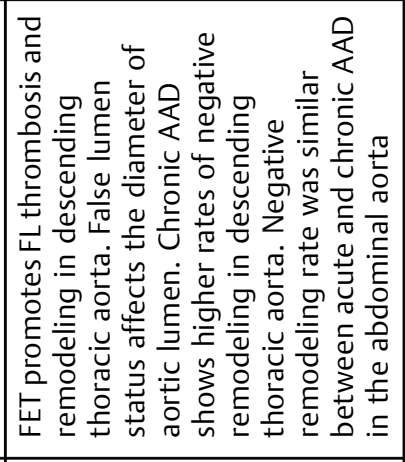 & 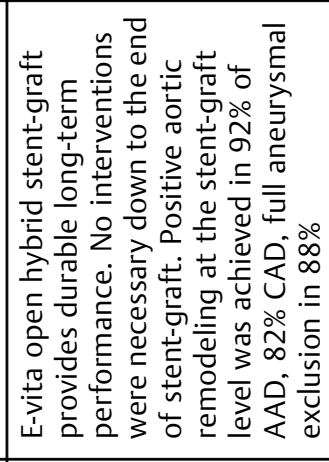 & 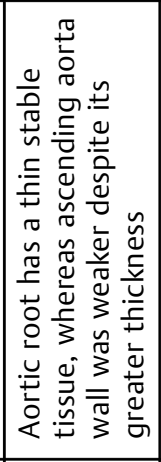 & 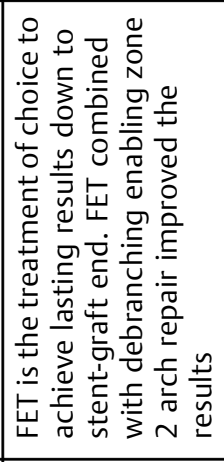 \\
\hline 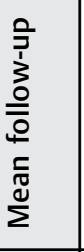 & 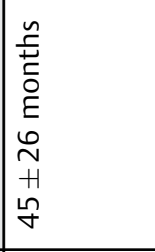 & 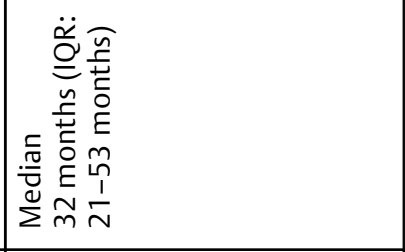 & 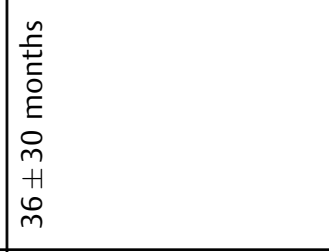 & $\Sigma$ & $\Sigma$ \\
\hline 产 & 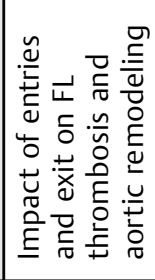 & 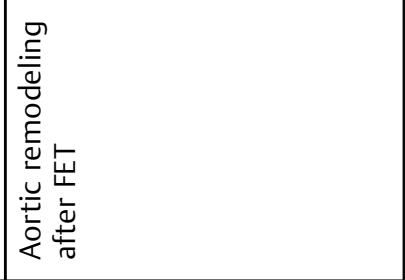 & 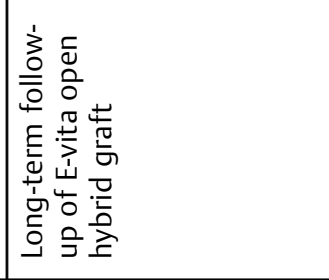 & 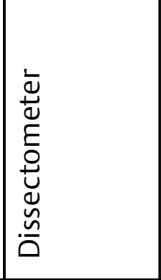 & 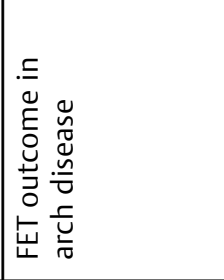 \\
\hline 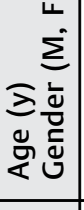 & 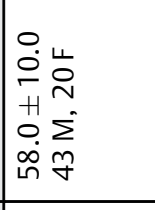 & 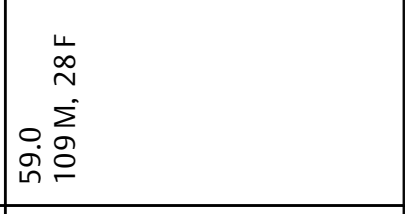 & 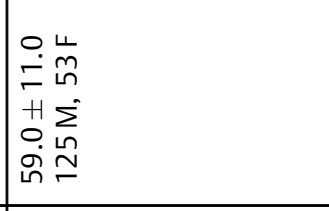 & 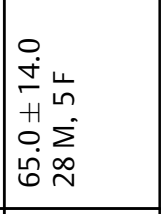 & 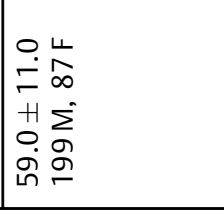 \\
\hline $\begin{array}{l}\text { E } \\
\frac{\tilde{N}}{n} \\
\frac{0}{n} \\
\frac{E}{n} \\
\text { ñ }\end{array}$ & 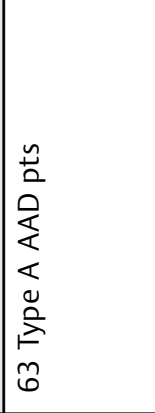 & 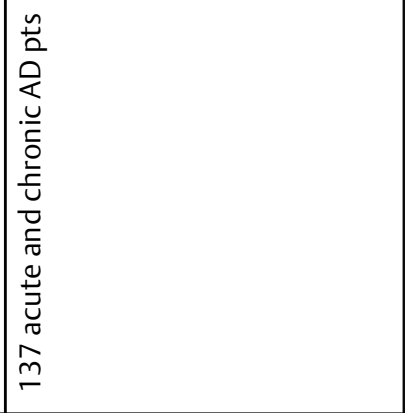 & 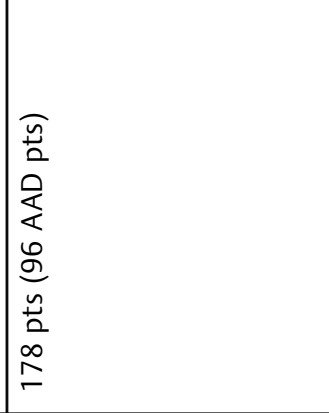 & 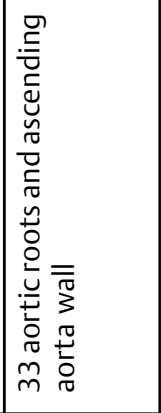 & 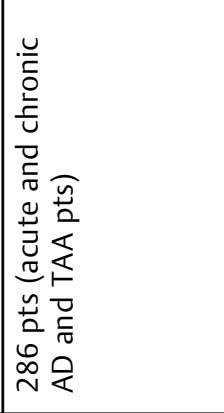 \\
\hline 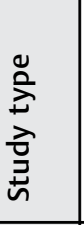 & 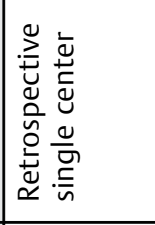 & 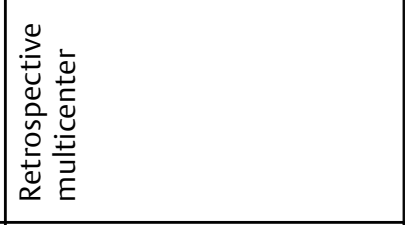 & 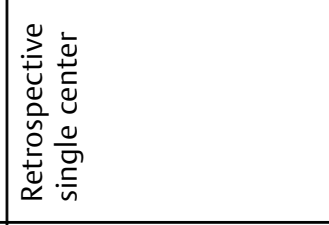 & 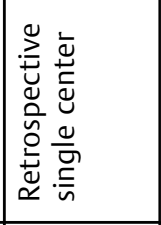 & 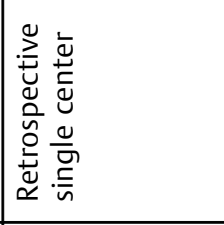 \\
\hline 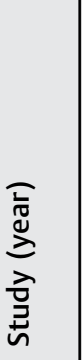 & 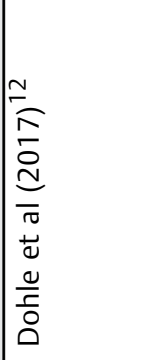 & 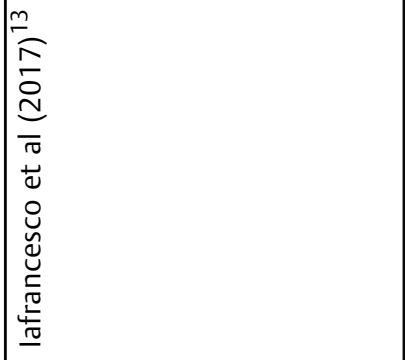 & 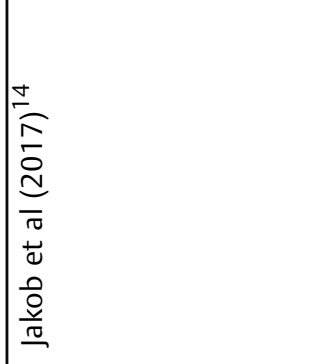 & 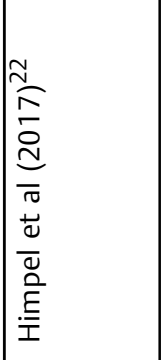 & 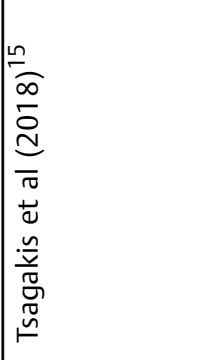 \\
\hline
\end{tabular}




\begin{tabular}{|c|c|c|c|c|c|c|c|c|c|c|c|c|}
\hline 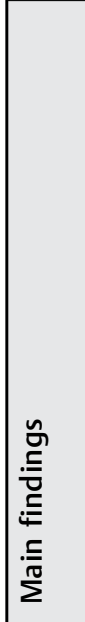 & 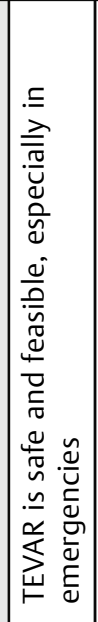 & 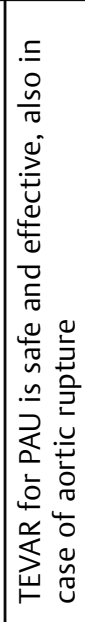 & 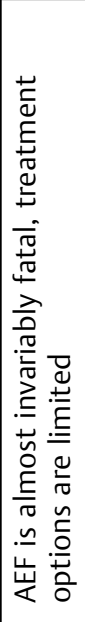 & 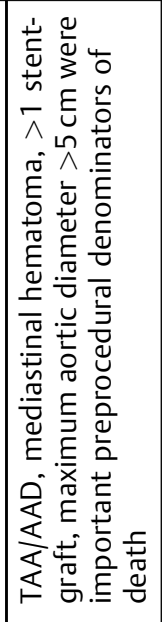 & 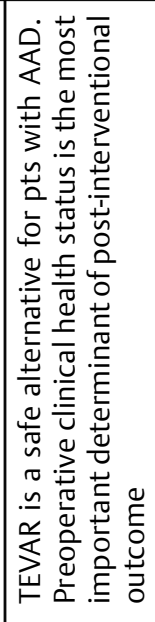 & 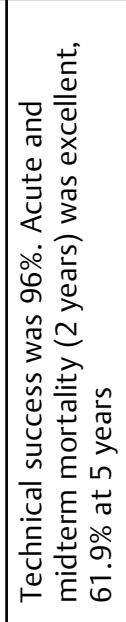 & 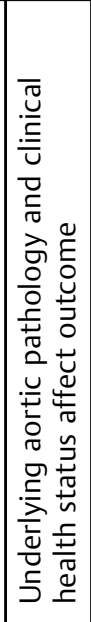 & 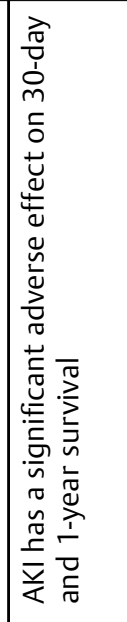 & 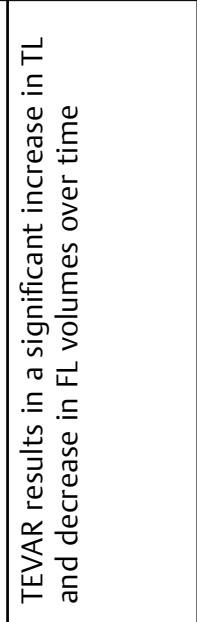 & 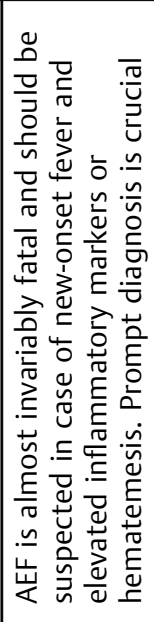 & 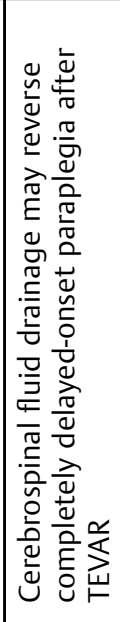 & 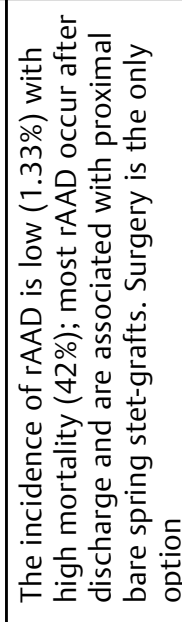 \\
\hline 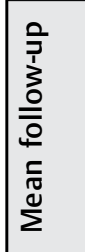 & 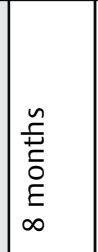 & 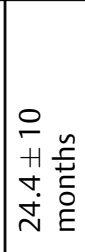 & 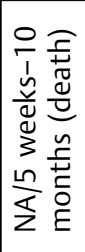 & 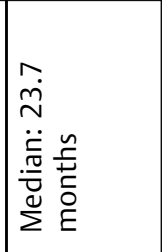 & 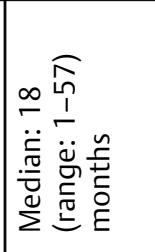 & 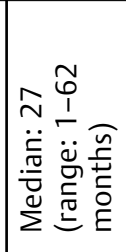 & 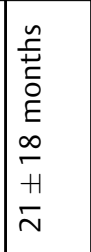 & 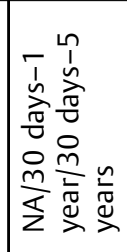 & 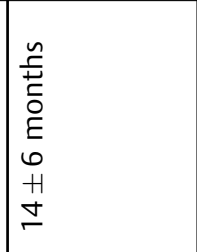 & 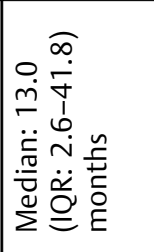 & ₹ & 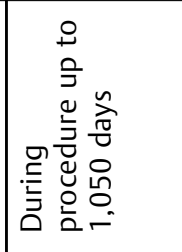 \\
\hline$\frac{.}{0}$ & 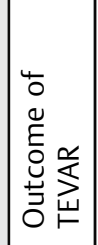 & 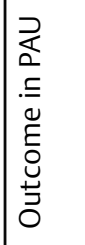 & 岀 & 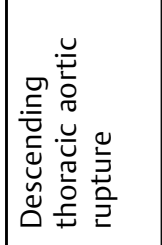 & 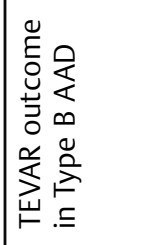 & 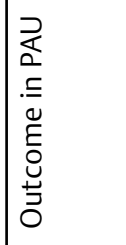 & 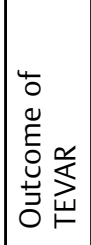 & 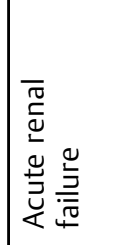 & 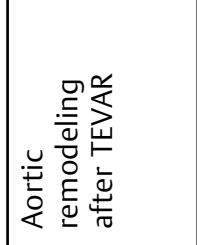 & | & 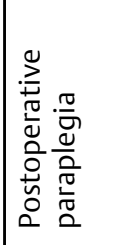 & 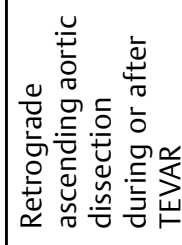 \\
\hline 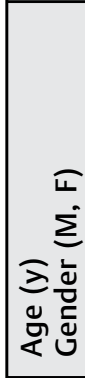 & 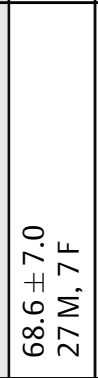 & 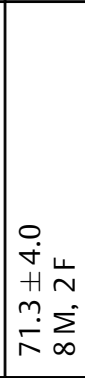 & 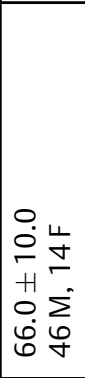 & 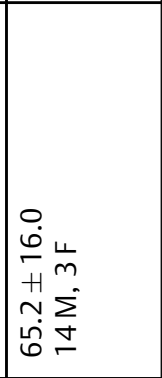 & 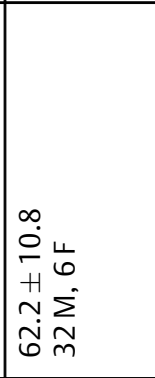 & 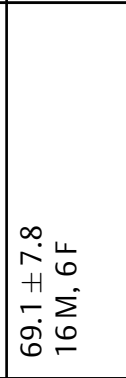 & 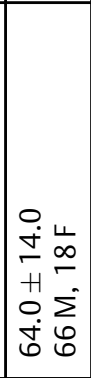 & 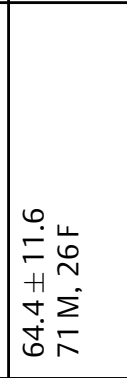 & 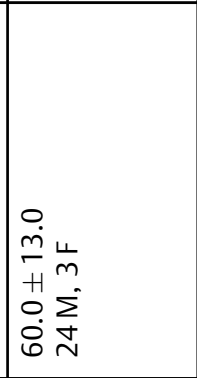 & 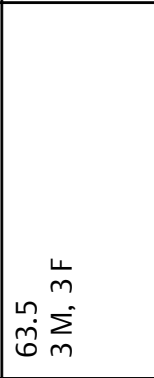 & 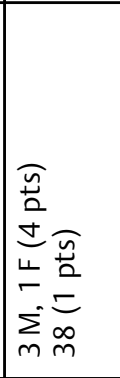 & 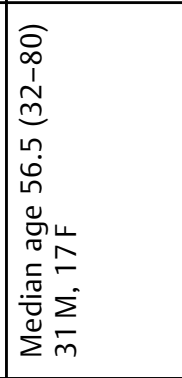 \\
\hline $\begin{array}{l}\text { อ } \\
\stackrel{\tilde{N}}{n} \\
\frac{0}{0} \\
\frac{0}{E} \\
\tilde{n}\end{array}$ & 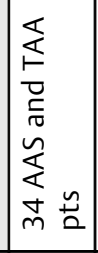 & 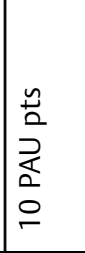 & 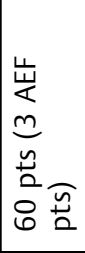 & 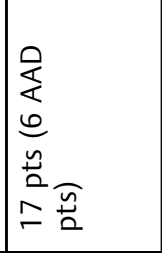 & 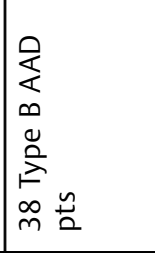 & 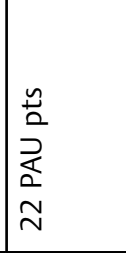 & 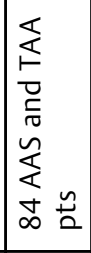 & 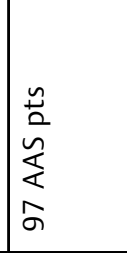 & 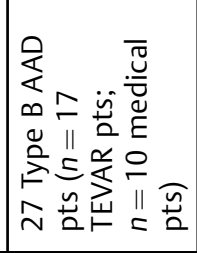 & 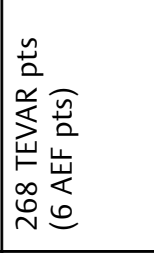 & 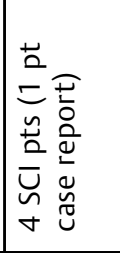 & 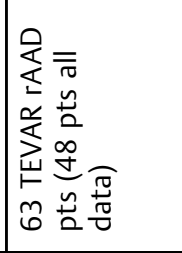 \\
\hline 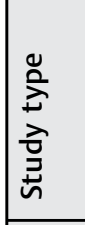 & 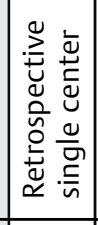 & 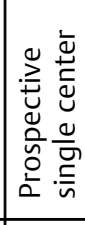 & 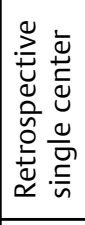 & 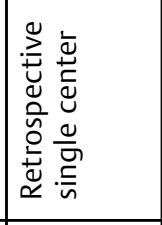 & 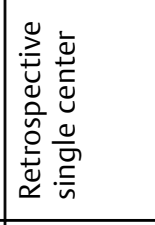 & 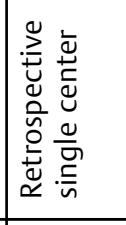 & 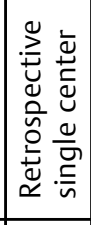 & 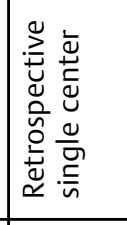 & 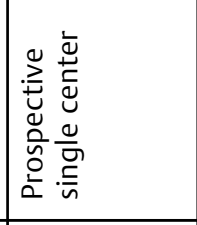 & 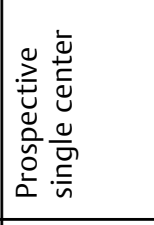 & 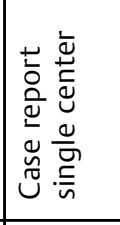 & 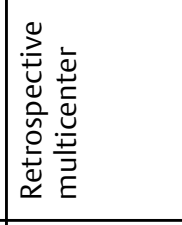 \\
\hline 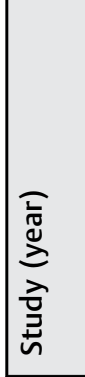 & 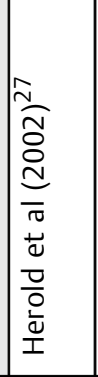 & 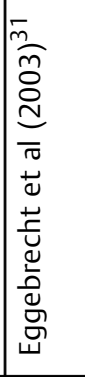 & 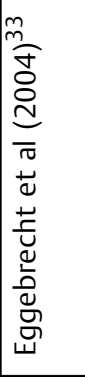 & 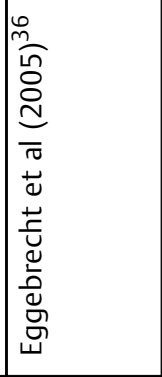 & 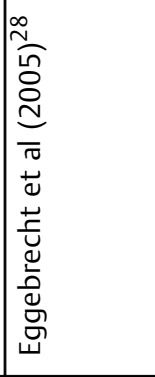 & 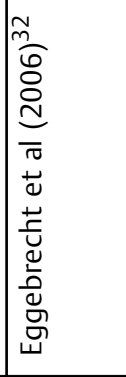 & 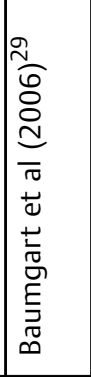 & 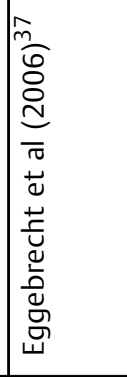 & 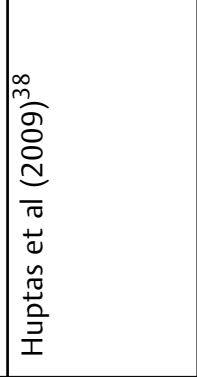 & 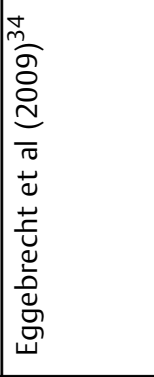 & 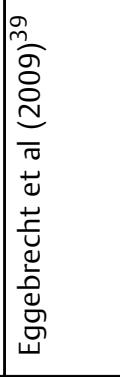 & 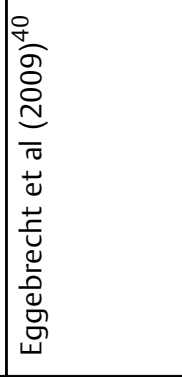 \\
\hline
\end{tabular}




\begin{tabular}{|c|c|c|c|c|c|}
\hline 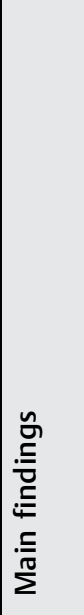 & 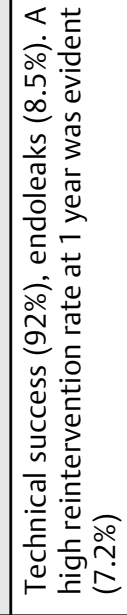 & 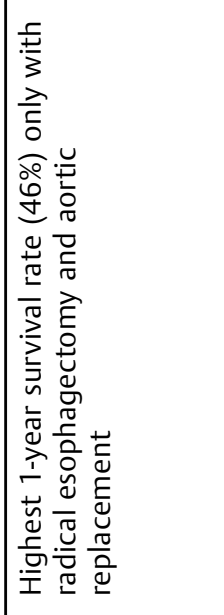 & 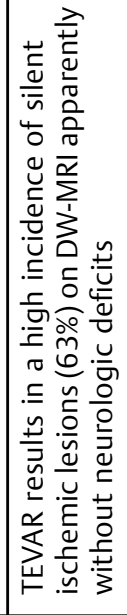 & 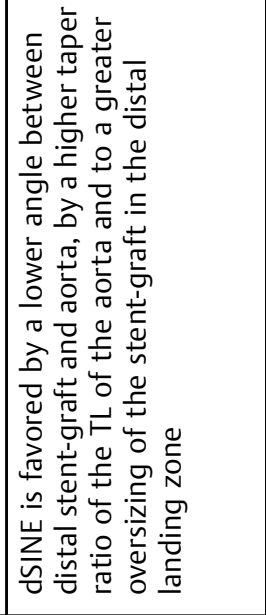 & 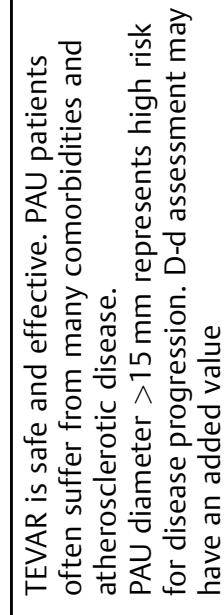 \\
\hline 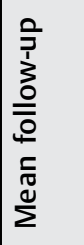 & 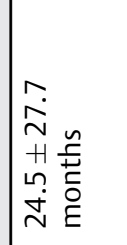 & 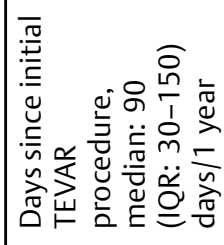 & 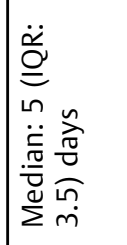 & 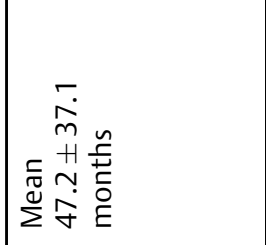 & 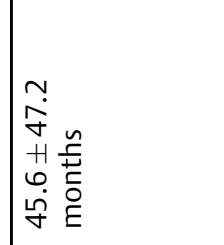 \\
\hline$\frac{\sqrt{0}}{0}$ & 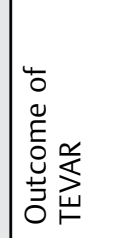 & 岀 & 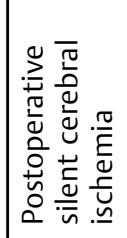 & 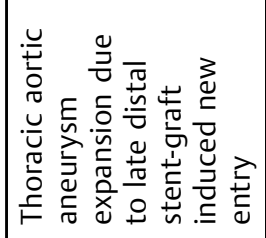 & 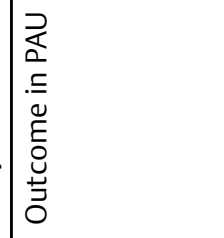 \\
\hline 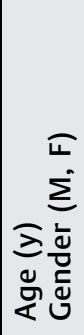 & 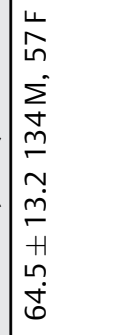 & 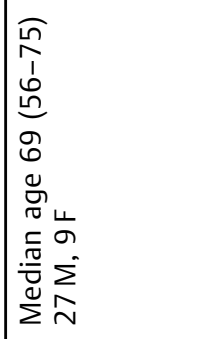 & 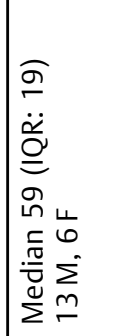 & 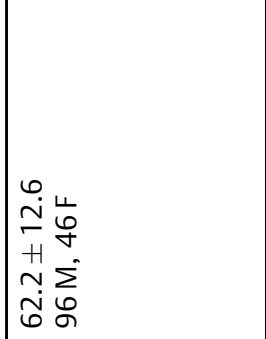 & 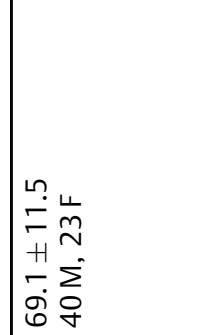 \\
\hline 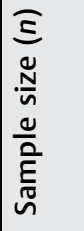 & 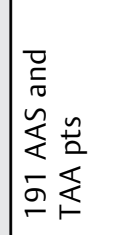 & 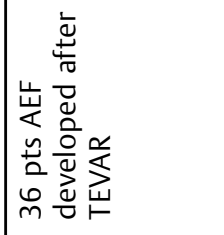 & 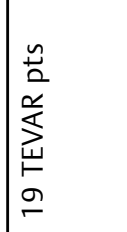 & 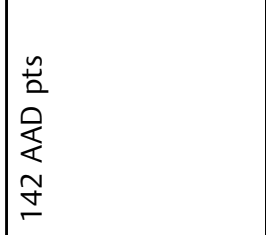 & 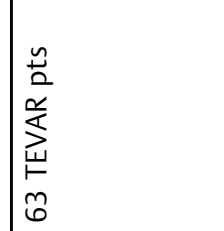 \\
\hline 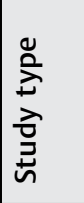 & 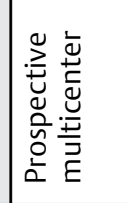 & 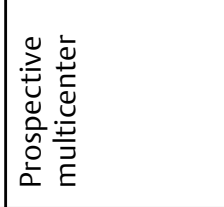 & 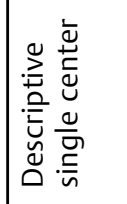 & 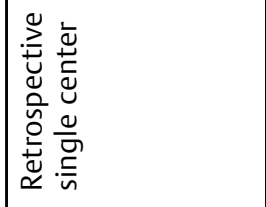 & 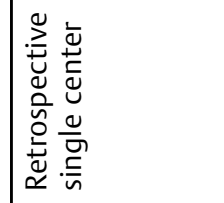 \\
\hline 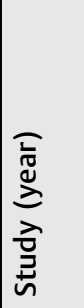 & 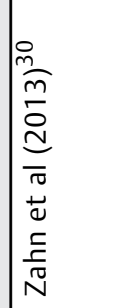 & 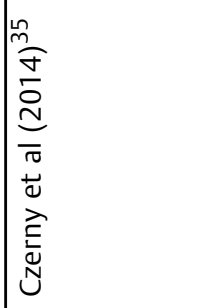 & 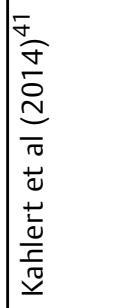 & 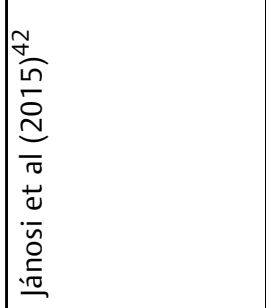 & 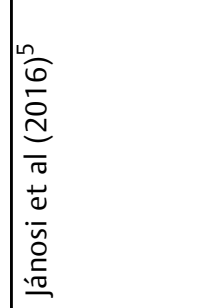 \\
\hline
\end{tabular}

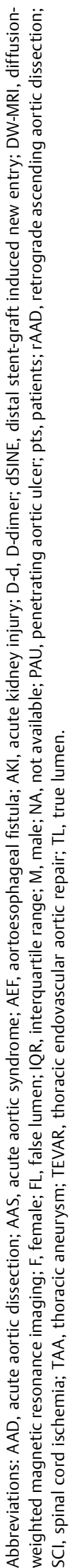




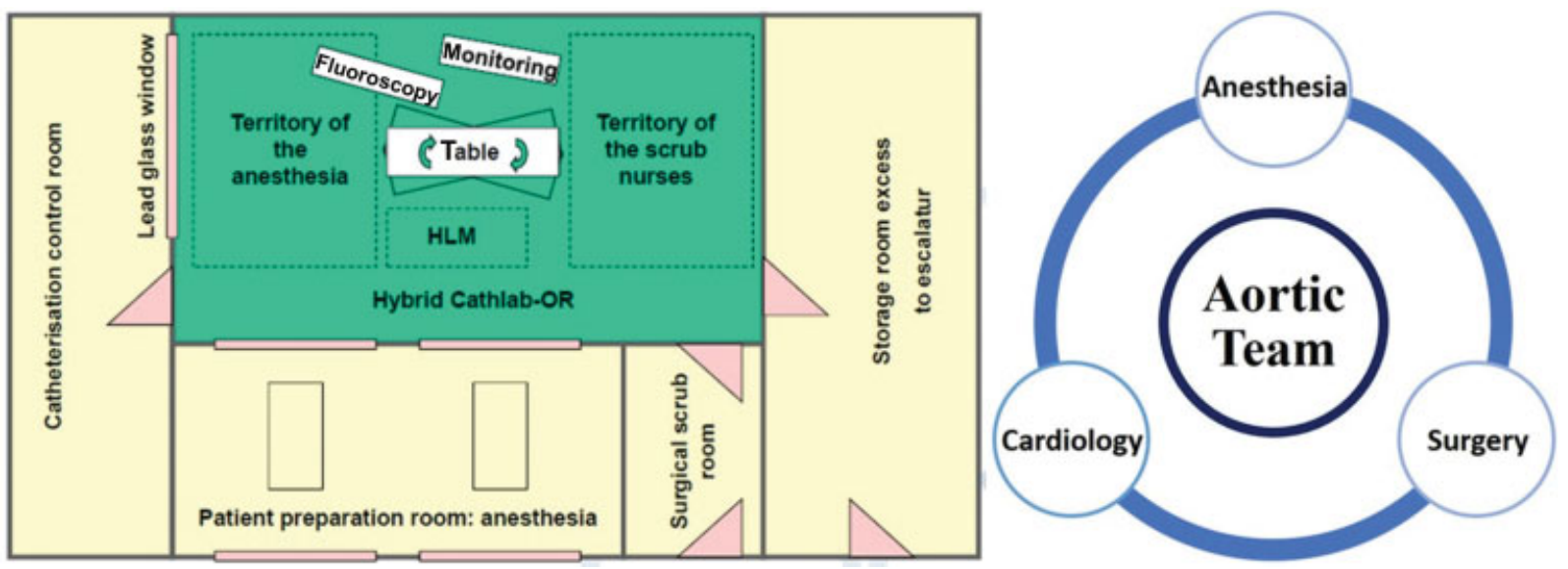

Fig. 1 Essen hybrid room concept: invasive diagnostics and endovascular +open surgery. HLM, heart-lung machine; OR, operating room. ${ }^{26}$

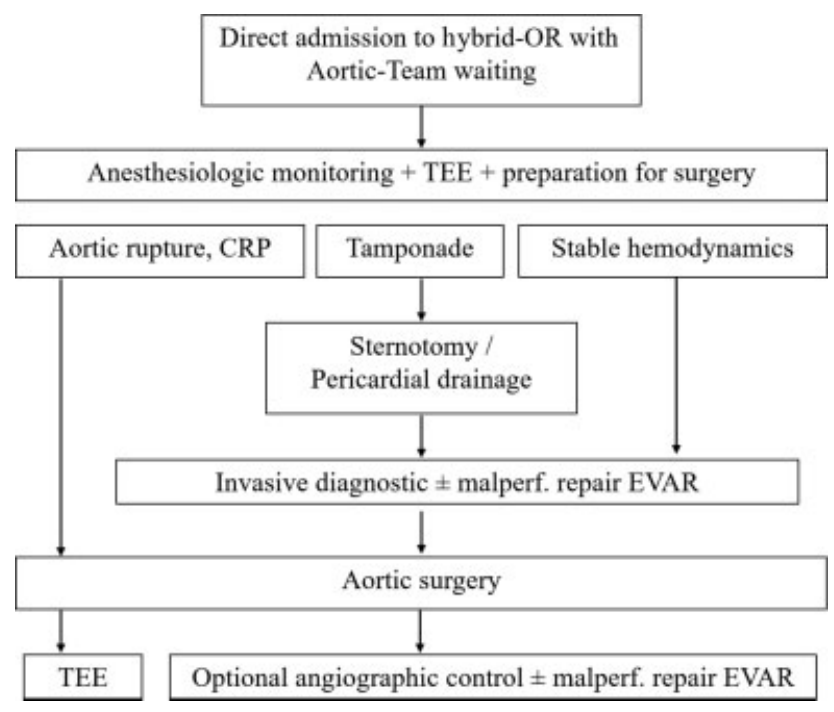

Fig. 2 Essen algorithm for hybrid operating room concept for acute Type A aortic dissection management. CRP, C-reactive protein; EVAR, endovascular aneurysm repair; OR, operating room; TEE,

transesophageal echocardiography. Image courtesy: Tsagakis et al. ${ }^{26}$

and the surgeon is standing by to intervene immediately in case of a complication.

An elaborate malperfusion regimen has been developed over the past 10 years enabling decision-making, for example, whether or not to treat severe visceral malperfusion prior to surgery or afterward (-Fig. 3). ${ }^{26}$ Thus, it becomes possible to restore end-organ perfusion prior to surgery by endovascular means. In case of prolonged ( $>6$ hours) total or subtotal malperfusion with elevation of liver enzymes and lactate and/or in symptomatic patients, an intensive care unit controlled delay to definitive surgical treatment of the underlying thoracic aortic pathology can be chosen in hemodynamically stable patients under continuous TEE control. $^{48,49}$

The Essen hybrid OR concept demonstrates the possibility of individualized management for Type A AAD based on the Penn classification, because advanced diagnostics and therapeutics can be performed without delay in a single clinical

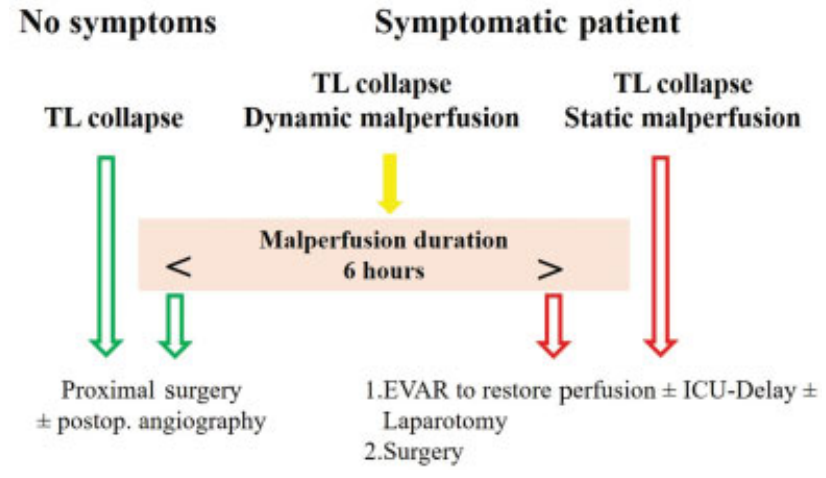

Fig. 3 Essen algorithm for visceral malperfusion. Treatment planning in the hybrid operating room. EVAR, endovascular aneurysm repair; ICU, intensive care unit; TL, true limen. ${ }^{26}$

venue. ${ }^{50,51}$ The hybrid OR concept and associated developments resulting from its improved capabilities for diagnostics, intervention, and differentiated surgical options enables individualized, patient-oriented optimal treatment options. The authors believe that the associated clinical outcome is better compared with the conventional approach, though not yet reaching statistical significance. ${ }^{26,51}$

\section{Management of Type B Acute Aortic Syndromes}

Definitive management of uncomplicated Type B AAD consists of close surveillance (clinical and imaging) and aggressive medical therapy (in-hospital mortality $\sim 11 \%)^{1,3,4}$ Nowadays, according to the $\operatorname{ESC}^{1}$ and more recent European Society for Vascular Surgery (ESVS) ${ }^{52}$ guidelines, TEVAR is recommended as the therapy of choice in complicated Type $B$ $A A D,{ }^{1}$ defined by persistent or recurrent pain, uncontrolled hypertension despite full medication, early aortic expansion, malperfusion, and signs of rupture (hemothorax, increasing periaortic, and mediastinal hematoma). ${ }^{1}$ Optimal positioning of stent-grafts leads to thrombus formation in the false lumen and initiates aortic healing with complete restoration of normal anatomy in many cases, in case of continuing 
patency of the false lumen, slow but continuous aortic enlargement can result. ${ }^{38}$ Implantation of additional bare metal stents was introduced as "PETTICOAT" stenting by Nienaber et al. ${ }^{53}$ In parallel, the later called "STABILISE" technique has been introduced by Hofferberth et $\mathrm{al}^{54}$ in the first decade of this century, using a balloon fracturing method within the distal portion of the covered descending stent-graft to rupture the separating lamella, protected by noncovered stents down to aortic bifurcation. Thus, perfect relamination of the stented aorta could be achieved. ${ }^{54}$ It should also be highlighted that TEVAR may induce a positive aortic remodeling at the level of the thoracic aorta, with continuous gain in true lumen volume over time, as well as progressive reduction of false lumen volume, in contrast to medically treated uncomplicated Type B AAD patients. This finding has relevant clinical implications implying potential therapeutic benefit of TEVAR over medical therapy alone in uncomplicated Type B dissection which warrants further randomized studies. ${ }^{55,56}$ Thus, all patients with suspected or proven Type B AAD are treated in the hybrid room initially, and in ensuing diagnostic and therapeutic sessions, as well. In case of persisting true lumen compression, noncovered stents are placed downstream in addition to the covered stent at the descending aortic position. Finally, open surgery may be considered a treatment option in the case of complicated Type B AAS, not suitable to TEVAR (in-hospital mortality rate $\sim 30 \%)^{1,3,4}$

\section{Studies Addressing Thoracic Endovascular Aortic Repair Outcome in Patients with Type B Acute Aortic Syndromes}

Already, the first case of Type B AAD which was detected by TEE opened the question of further therapy. Most striking was the imaging of the entry tear at the descending aorta just distally to the origin of the subclavian artery which had not been reported previously. ${ }^{57}$ Closure of the tear seemed to be the logical consequence of this finding, but at that time, few surgeons were available who were experienced in aortic surgery. At that time, paraplegia after surgery was observed quite often. In this regard, feasibility and safety of TEVAR was assessed by Eggebrecht et $\mathrm{al}^{28}$ in 38 aortic dissection patients, with excellent overall in-hospital survival (89\%), 30-day mortality (97.4\%), 1-year (80.4\%), 2-year (73.2\%), and 4-year survival (54.9\%). Intimal entry could be sealed in all cases and, notably, a preoperative poor clinical health status was the most important determinant of postinterventional mortality. Excellent technical success rates (>90\%) were confirmed also by other single-center and multicenter reports. $^{27,29,30}$

Zahn et $\mathrm{al}^{30}$ enrolled 191 patients undergoing TEVAR including 104 Type B AAD, 91 thoracic aortic aneurysm, 20 Type $B$ acute intramural hematoma, 16 Type $B$ acute penetrating aortic ulcer (PAU), and 6 traumatic aortic ruptures. A high technical success rate (92.1\%) along with acceptable inhospital complication rates were registered (14.1\%; 15 patients presented endoleaks [Types I-III], 7 patients stroke, and 3 paraplegia; only 6 patients needed a reintervention). The in-hospital mortality was $5.5 \%$. The 1 -year reinterven- tion and mortality rates were 7.2 and $11.4 \%$, respectively (-Supplementary Fig. S4).

Jánosi et $\mathrm{al}^{5}$ analyzed 63 patients with complicated PAU (42 symptomatic and 22 with rupture) who underwent TEVAR (-Supplementary Fig. S5A). Among these patients, 33 received TEVAR within 14 days of diagnosis, while the other 30 were treated with an average interval between diagnosis and intervention of $40 \pm 39$ days. An excellent technical success rate (98.4\%) along with an in-hospital mortality of $7.9 \%$ was observed. Long-term overall survivals estimated at 1,5 , and 10 years were $88.4,82.2$, and $65.7 \%$, respectively. During follow-up, $19.0 \%$ of the patients needed a secondary intervention due to new complications or late endoleaks. Furthermore, there was no significant difference in long-term survival between patients who had undergone TEVAR for PAU and the historical cohort of patients treated with TEVAR for classic false lumen Type B AAD (-Supplementary Fig. S5B). ${ }^{5}$ Interestingly, a PAU diameter of $>15 \mathrm{~mm}$ and higher D-dimer levels appear to play a role in the identification of patients at risk and with disease progression. $^{5}$

\section{Thoracic Endovascular Aortic Repair-Related Complications}

Several important complications related to the procedure may affect the postoperative course of TEVAR patients and should therefore be promptly suspected and adequately managed by clinicians.

- Acute kidney injury: postprocedural acute kidney injury (AKI) is a complication related to catheter-based interventional procedures that use intra-arterial contrast agents. The prevalence of AKI, defined as an increase $\geq 25 \%$ and/or $0.5 \mathrm{mg} / \mathrm{dL}$ in preprocedural serum creatinine at 48 hours after TEVAR, has been reported in $38.9 \%{ }^{58}$ and $34 \%^{37}$ of patients with AAS. Development of AKI was closely related to preoperative hemoglobin levels and postoperative hemoglobin drop, and these factors were independent predictors of in-hospital mortality. Additionally, malperfusion at admission, higher loads of contrast medium, and diabetes mellitus were important risk factors for AKI. Therefore, the use of an isoosmolar contrast medium, as well as measures aiming at preventing procedure-related bleeding and access site complications and careful monitoring of fluid status to avoid excessive hemodilution should be pursued. The prognostic impact of AKI on follow-up mortality remains to be clarified.

- Postimplantation syndrome (PIS): TEVAR induces elevations of inflammatory and coagulative biomarkers (white blood cells, C-reactive protein, fibrinogen, and D-dimer). ${ }^{59}$ In $\sim 16 \%$ of patients, the inflammatory response leads to clinical manifestations, including fever with negative blood cultures sometimes accompanied by back or thoracic pain within 48 to 72 hours after TEVAR. This is known as PIS. ${ }^{60}$ Diagnostic criteria of PIS include at least two of the four systemic inflammatory response syndrome criteria (fever $>38^{\circ} \mathrm{C}$, white blood cell count $>12,000 / \mu \mathrm{L})$. An increase in C-reactive protein $>10 \mathrm{mg} / \mathrm{dL}$ 
may be an additional criterion with important prognostic implications in addition to interleukin-6 which seems to be PIS specific (-Supplementary Fig. S6). ${ }^{61}$

Interestingly, although PIS did not affect in-hospital outcome, it was associated with higher rates of major adverse events (aortic rupture, endoleak Type 1, and reintervention), but not of mortality at 4-year follow-up. ${ }^{61}$ However, the role of corticosteroids as therapeutic agents aiming at preventing complications during follow-up should be validated in future studies.

- Aortoesophageal fistula: aortoesophageal fistula (AEF) is a rare and dreaded complication following TEVAR which requires a high index of suspicion for a prompt diagnosis. The reported prevalence is $1.9 \%$ and it is characterized by new-onset fever with elevated inflammatory biomarkers (81\%), hematemesis (53\%), and shock (22\%). ${ }^{33,34}$ Prompt diagnosis with CT (new mediastinal mass) or esophagogastroduodenoscopy (visualization of the stent-graft) is of paramount importance (-Supplementary Fig. S7). ${ }^{34}$ If medically treated, this condition is invariably fatal, whereas combined radical esophagectomy and aortic replacement has the highest 1-year survival (43\%). ${ }^{35}$

- Symptomatic spinal cord ischemia: its prevalence is up to $5 \%$ and it occurs more frequently in emergency scenarios. Prolonged intraoperative hypotension combined with simultaneous closure of at least two of four independent spinal cord supplying territories (subclavian, intercostal, lumbar, and hypogastric) had the strongest association with symptomatic spinal cord ischemia. This finding emphasizes the importance of preserving the left subclavian artery during TEVAR to maintain posterior cerebellar perfusion. In contrast, extensive coverage of intercostal arteries by a thoracic stent-graft alone seems not relevant. $^{62}$ The onset of symptomatic spinal cord ischemia may occur up to several hours after TEVAR procedures in up to $50 \%$ of cases. In addition to augmentation of mean arterial pressure, cerebrospinal fluid drainage was effective in complete reversal of patient's symptoms but can lead to serious complications such as spinal headache and epidural/subdural hematoma.

- Silent cerebral ischemia: the risk of clinically apparent periprocedural stroke after TEVAR has been reported between 2 and $6 \%$ and is related to intravascular manipulation of guidewires, catheters, and delivery systems, with subsequent dislodgement of atherosclerotic plaque debris. However, new silent cerebral lesions have been identified in $63 \%$ of patients in a diffusion-weighted magnetic resonance imaging (MRI) study, with no differences according to the indication for TEVAR, overstenting of the left subclavian artery (provided the exclusion of patients with left dominant vertebral artery), stent landing zone, and previous aortic surgery. Further studies are needed to identify patient- and procedure-related risk factors for cerebral embolism during TEVAR. ${ }^{41}$
- Retrograde ascending aortic dissection: although the prevalence of this potentially lethal complication is very low (1.33\%), retrograde ascending aortic dissection (RAAD) is associated with high mortality (42\%). It can occur preoperatively (21\%), during TEVAR (15\%), or more frequently during follow-up (65\%). In the majority of cases, RAAD is related to the use of stent-grafts with bare proximal springs. Direct evidence of induced stent-graft has been confirmed injury at surgery or necropsy. Less frequently RAAD may be attributed to disease progression or manipulation of guidewires and sheaths during the procedure (-Supplementary Fig. S8). ${ }^{40}$ Presentation symptoms include chest pain, syncope, or stroke, but patients can be asymptomatic in $25 \%$ of cases. Surgical repair is the only option if dissection is related to stent-graft injury, whereas a conservative treatment may be feasible if dissection is wire related. ${ }^{40}$

- Distal stent-graft induced new entry: the reported prevalence is 6.3\%; this can occur up to 6 years after the index procedure, and patients are often asymptomatic. Once the diagnosis is made on CT angiography, close follow-up at every 3 months should be recommended to evaluate the progression and formation of the distal aneurysm caused by expansion of the false lumen. Indications for reintervention include persistent enlargement of the false lumen, symptomatic state, and signs of rupture. Patients with greater oversizing of the stent-graft at the distal landing zone, as well as those with a more acute angle between the bottom edge of the stent-graft and aorta were at higher risk of stentgraft induced new entry. Therefore, especially in older dissection, distal oversizing should be kept to a minimum or avoided, or tapered stent-grafts may be preferred. Technical success of reintervention was $100 \%{ }^{42}$

\section{Long-Term Follow-up Clinic}

AAS is a life-long medical entity involving the entire aorta and its branches (holistic view) implying a substantial risk for redissection, aneurysm formation, and rupture. ${ }^{1}$ Thus, all patients affected by AAS should undergo close clinical and imaging follow-up (before discharge and at 1, 3, 6, and 12 months, and annually thereafter [biannually in the case of very stable patients]). Although CT is the most used imaging technique, MRI should be preferred (especially in young patients; being ionizing radiation free). In Essen, a weekly outpatient clinic is held and new patients are screened, as well as all patients having undergone aortic surgery are seen annually or biannually thereafter, including imaging. Thus, exact knowledge of the patients' status and their aorta is available to determine whether or not additional therapeutic steps are necessary. ${ }^{63}$ Optimal blood pressure and heart rate control (blood pressure $<120 / 80 \mathrm{~mm} \mathrm{Hg}$, heart rate $<60 \mathrm{bpm}$ ) represent a cornerstone of medical treatment (first line: long-acting $\beta$-blockers and second line: angiotensin-converting enzyme inhibitors or angiotensin receptor blockers). Secondary prevention measures tailored on an individual patient basis should be implemented to reduce the burden of cardiovascular risk factors. ${ }^{1}$ To ensure a perfect follow-up, the Aortic Center in 
Essen organized regular patient visits; patients are seen by members of the cardiology and cardiovascular surgery team, including comprehensive CT or MRI follow-up imaging but also addressing blood pressure control and medical events.

\section{Conclusion}

The integrated multidisciplinary endovascular and surgical approach to AAS, along with the design and implementation of the hybrid OR concept, have represented a "Rosetta Stone" of AAS therapeutic interventions, paving the way for investigators to follow. In this regard, endovascular treatment of clinically stable Type B AAS remains an open question to be answered by future studies. Serologic and imaging biomarkers may play a key role on early detection of unstable disease in otherwise clinically stable patients.

\section{Funding}

None.

\section{Conflict of Interest}

The authors declare no conflict of interest related to this article.

\section{Acknowledgments}

None.

\section{References}

1 Erbel R, Aboyans V, Boileau C, et al; ESC Committee for Practice Guidelines The Task Force for the Diagnosis and Treatment of Aortic Diseases of the European Society of Cardiology (ESC) 2014 ESC Guidelines on the diagnosis and treatment of aortic diseases: Document covering acute and chronic aortic diseases of the thoracic and abdominal aorta of the adult. Eur Heart J 2014;35 (41):2873-2926

2 Bossone E, Gorla R, Ranieri B, Russo V, Jakob H, Erbel R. Initiating a new era of cardiovascular diagnosis and therapy in acute aortic syndromes: the Mainz-Essen experience (Part I) - imaging and biomarkers. Aorta (Stamford) 2021 (e-pub ahead of print). DOI: 10.1055/s-0041-1730295

3 Evangelista A, Isselbacher EM, Bossone E, et al; IRAD Investigators. Insights from the International Registry of Acute Aortic Dissection: a 20-year experience of collaborative clinical research. Circulation 2018;137(17):1846-1860

4 Pape LA, Awais M, Woznicki EM, et al. Presentation, diagnosis, and outcomes of acute aortic dissection: 17-year trends from the International Registry of Acute Aortic Dissection.J Am Coll Cardiol 2015;66(04):350-358

5 Jánosi RA, Gorla R, Tsagakis K, et al. Thoracic endovascular repair of complicated penetrating aortic ulcer: an 11-year single-center experience. J Endovasc Ther 2016;23(01):150-159

6 Jakob H, Tsagakis K, Tossios P, et al. Combining classic surgery with descending stent grafting for acute DeBakey type I dissection. Ann Thorac Surg 2008;86(01):95-101

7 Tsagakis K, Pacini D, Di Bartolomeo R, et al. Multicenter early experience with extended aortic repair in acute aortic dissection: is simultaneous descending stent grafting justified? J Thorac Cardiovasc Surg 2010;140(6, suppl):S116-S120, discussion S142-S146

8 Pacini D, Tsagakis K, Jakob $\mathrm{H}$, et al. The frozen elephant trunk for the treatment of chronic dissection of the thoracic aorta: a multicenter experience. Ann Thorac Surg 2011;92(05): 1663-1670, discussion 1670
9 Weiss G, Tsagakis K, Jakob H, et al. The frozen elephant trunk technique for the treatment of complicated type B aortic dissection with involvement of the aortic arch: multicentre early experience. Eur J Cardiothorac Surg 2015;47(01):106-114, discussion 114

10 Dohle DS, Tsagakis K, Janosi RA, et al. Aortic remodelling in aortic dissection after frozen elephant trunk. Eur J Cardiothorac Surg 2016;49(01):111-117

11 Leontyev S, Tsagakis K, Pacini D, et al. Impact of clinical factors and surgical techniques on early outcome of patients treated with frozen elephant trunk technique by using EVITA open stent-graft: results of a multicentre study. Eur J Cardiothorac Surg 2016;49 (02):660-666

12 Dohle DS, Jakob H, Schucht R, et al. The impact of entries and exits on false lumen thrombosis and aortic remodelling. Eur J Cardiothorac Surg 2017;52(03):508-515

13 Iafrancesco M, Goebel N, Mascaro J, et al; International E-vita Open Registry Group. Aortic diameter remodelling after the frozen elephant trunk technique in aortic dissection: results from an international multicentre registry. Eur J Cardiothorac Surg 2017;52(02):310-318

14 Jakob H, Dohle D, Benedik J, et al. Long-term experience with the E-vita open hybrid graft in complex thoracic aortic disease. Eur J Cardiothorac Surg 2017;51(02):329-338

15 Tsagakis K, Wendt D, Dimitriou AM, et al. The frozen elephant trunk treatment is the operation of choice for all kinds of arch disease. J Cardiovasc Surg (Torino) 2018;59(04):540-546

16 Tsagakis K, Kamler M, Kuehl H, et al. Avoidance of proximal endoleak using a hybrid stent graft in arch replacement and descending aorta stenting. Ann Thorac Surg 2009;88(03): 773-779

17 Tsagakis K, Pacini D, Di Bartolomeo R, et al. Arch replacement and downstream stent grafting in complex aortic dissection: first results of an international registry. Eur J Cardiothorac Surg 2011; 39(01):87-93, discussion 93-94

18 Jakob H, Dohle DS, Piotrowski J, et al. Six-year experience with a hybrid stent graft prosthesis for extensive thoracic aortic disease: an interim balance. Eur J Cardiothorac Surg 2012;42(06): 1018-1025

19 Tsagakis K, Dohle D, Benedik J, Lieder H, Jakob H. Overall Essen's experience with the E-vita open hybrid stent graft system and evolution of the surgical technique. Ann Cardiothorac Surg 2013; 2(05):612-620

20 Benedik J Jr., Azhari P, Tsagakis K, et al. Dissectometer - a new device for tensile strength testing of the vascular wall. Minim Invasive Ther Allied Technol 2012;21(05):329-334

21 Pilarczyk K, Tsagakis K, Thielmann M, et al. Detection of aortic wall instability with the new dissectometer: Correlation with histological findings. Minim Invasive Ther Allied Technol 2015;24 (04):233-241

22 Himpel L, Pilarczyk K, Wahbi A, et al. Role of regional aortic wall properties in the pathogenesis of thoracic aortic dissection. Scand Cardiovasc J 2017;51(01):35-39

23 Jakob H, Tsagakis K. DeBakey type I dissection: when hybrid stentgrafting is indicated? J Cardiovasc Surg (Torino) 2010;51(05): 633-640

24 Tsagakis K, Kamler M, Benedik J, Jakob H. Angioscopy-a valuable tool in guiding hybrid stent grafting and decision making during type A aortic dissection surgery. Eur J Cardiothorac Surg 2010;38 (04):507-509

25 Tsagakis K, Tossios P, Kamler M, et al. The DeBakey classification exactly reflects late outcome and re-intervention probability in acute aortic dissection with a slightly modified type II definition. Eur J Cardiothorac Surg 2011;40(05):1078-1084

26 Tsagakis K, Konorza T, Dohle DS, et al. Hybrid operating room concept for combined diagnostics, intervention and surgery in acute type A dissection. Eur J Cardiothorac Surg 2013;43(02): 397-404 
27 Herold U, Piotrowski J, Baumgart D, Eggebrecht H, Erbel R, Jakob H. Endoluminal stent graft repair for acute and chronic type B aortic dissection and atherosclerotic aneurysm of the thoracic aorta: an interdisciplinary task. Eur J Cardiothorac Surg 2002;22(06): 891-897

28 Eggebrecht H, Herold U, Kuhnt O, et al. Endovascular stent-graft treatment of aortic dissection: determinants of post-interventional outcome. Eur Heart J 2005;26(05):489-497

29 Baumgart D, Eggebrecht $\mathrm{H}$, Herold $\mathrm{U}$, et al. Underlying aortic pathology and clinical health status determine success of endovascular stent-grafting for descending thoracic aortic disease. Catheter Cardiovasc Interv 2006;67(04):527-534

30 Zahn R, Erbel R, Nienaber CA, et al. Endovascular aortic repair of thoracic aortic disease: early and 1-year results from a German multicenter registry. J Endovasc Ther 2013;20(03):265-272

31 Eggebrecht H, Baumgart D, Schmermund A, et al. Endovascular stent-graft repair for penetrating atherosclerotic ulcer of the descending aorta. Am J Cardiol 2003;91(09):1150-1153

32 Eggebrecht $\mathrm{H}$, Herold U, Schmermund A, et al. Endovascular stentgraft treatment of penetrating aortic ulcer: results over a median follow-up of 27 months. Am Heart J 2006;151(02):530-536

33 Eggebrecht H, Baumgart D, Radecke K, et al. Aortoesophageal fistula secondary to stent-graft repair of the thoracic aorta. J Endovasc Ther 2004;11(02):161-167

34 Eggebrecht $\mathrm{H}$, Mehta RH, Dechene A, et al. Aortoesophageal fistula after thoracic aortic stent-graft placement: a rare but catastrophic complication of a novel emerging technique. JACC Cardiovasc Interv 2009;2(06):570-576

35 Czerny M, Eggebrecht $\mathrm{H}$, Sodeck G, et al. New insights regarding the incidence, presentation and treatment options of aortooesophageal fistulation after thoracic endovascular aortic repair: the European Registry of Endovascular Aortic Repair Complications. Eur J Cardiothorac Surg 2014;45(03):452-457

36 Eggebrecht H, Schmermund A, Herold U, et al. Endovascular stentgraft placement for acute and contained rupture of the descending thoracic aorta. Catheter Cardiovasc Interv 2005;66(04):474-482

37 Eggebrecht H, Breuckmann F, Martini S, et al. Frequency and outcomes of acute renal failure following thoracic aortic stentgraft placement. Am J Cardiol 2006;98(04):458-463

38 Huptas S, Mehta RH, Kühl H, et al. Aortic remodeling in type B aortic dissection: effects of endovascular stent-graft repair and medical treatment on true and false lumen volumes. J Endovasc Ther 2009;16(01):28-38

39 Eggebrecht H, Böse D, Gasser T, et al. Complete reversal of paraplegia after thoracic endovascular aortic repair in a patient with complicated acute aortic dissection using immediate cerebrospinal fluid drainage. Clin Res Cardiol 2009;98(12):797-801

40 Eggebrecht H, Thompson M, Rousseau H, et al; European Registry on Endovascular Aortic Repair Complications. Retrograde ascending aortic dissection during or after thoracic aortic stent graft placement: insight from the European registry on endovascular aortic repair complications. Circulation 2009;120(11, suppl): S276-S281

41 Kahlert P, Eggebrecht H, Jánosi RA, et al. Silent cerebral ischemia after thoracic endovascular aortic repair: a neuroimaging study. Ann Thorac Surg 2014;98(01):53-58

42 Jánosi RA, Tsagakis K, Bettin M, et al. Thoracic aortic aneurysm expansion due to late distal stent graft-induced new entry. Catheter Cardiovasc Interv 2015;85(02):E43-E53

43 Jakob H, Tsagakis K, Dohle DS, et al. Hybrid room technology as a prerequisite for the modern therapy of aortic dissection [in German]. Herz 2011;36(06):525-530

44 Jakob H, Tsagakis K, Leyh R, Buck T, Herold U. Development of an integrated stent graft-dacron prosthesis for intended one-stage repair in complex thoracic aortic disease. Herz 2005;30(08): 766-768
45 Mestres CA, Tsagakis K, Pacini D, et al; IEOR Registry Group. Onestage repair in complex multisegmental thoracic aneurysmal disease: results of a multicentre study. Eur J Cardiothorac Surg 2013;44(05):e325-e331

46 Jakob H, Tsagakis K. International E-vita open registry. Ann Cardiothorac Surg 2013;2(03):296-299

47 Jakob H, Tsagakis K, Szabo A, Wiese I, Thielmann M, Herold U. Rapid and safe direct cannulation of the true lumen of the ascending aorta in acute type A aortic dissection. J Thorac Cardiovasc Surg 2007;134(01):244-245

48 Tsagakis K, Pizanis N, Kamler M, et al. ICU controlled delay for acute type a aortic dissection repair after intervention for total visceral malperfusion: a way out of a dilemma? Thorac Cardiovasc Surg 2008;56(05):298-300

49 Tsagakis K, Jánosi RA, Frey UH, et al. True lumen stabilization to overcome malperfusion in acute type I aortic dissection. Semin Thorac Cardiovasc Surg 2019;31(04):740-748

50 Augoustides JG, Geirsson A, Szeto WY, et al. Observational study of mortality risk stratification by ischemic presentation in patients with acute type A aortic dissection: the Penn classification. Nat Clin Pract Cardiovasc Med 2009;6(02):140-146

51 Augoustides JG, Patel PA, Savino JS, Bavaria JE. The heart team approach to acute type A dissection: a new paradigm in the era of the integrated Penn classification and the Essen concept. Eur J Cardiothorac Surg 2013;43(02):404-405

52 Riambau V, Böckler D, Brunkwall J, et al; Esvs Guidelines Committee. Editor's choice - management of descending thoracic aorta diseases: clinical practice guidelines of the European Society for Vascular Surgery (ESVS). Eur J Vasc Endovasc Surg 2017;53 (01):4-52

53 Nienaber CA, Kische S, Zeller T, et al. Provisional extension to induce complete attachment after stent-graft placement in type B aortic dissection: the PETTICOAT concept. J Endovasc Ther 2006; 13(06):738-746

54 Hofferberth SC, Nixon IK, Boston RC, McLachlan CS, Mossop PJ. Stent-assisted balloon-induced intimal disruption and relamination in aortic dissection repair: the STABILISE concept. J Thorac Cardiovasc Surg 2014;147(04):1240-1245

55 Nienaber CA, Kische S, Rousseau $\mathrm{H}$, et al; INSTEAD-XL trial. Endovascular repair of type $B$ aortic dissection: long-term results of the randomized investigation of stent grafts in aortic dissection trial. Circ Cardiovasc Interv 2013;6(04):407-416

56 Tadros RO, Tang GHL, Barnes HJ, et al. Optimal treatment of uncomplicated type B aortic dissection: JACC review topic of the week. J Am Coll Cardiol 2019;74(11):1494-1504

57 Börner N, Erbel R, Braun B, Henkel B, Meyer J, Rumpelt J. Diagnosis of aortic dissection by transesophageal echocardiography. Am J Cardiol 1984;54(08):1157-1158

58 Gorla R, Tsagakis K, Horacek M, et al. Impact of preoperative anemia and postoperative hemoglobin drop on the incidence of acute kidney injury and in-hospital mortality in patients with type B acute aortic syndromes undergoing thoracic endovascular aortic repair. Vasc Endovascular Surg 2017;51(03): 131-138

59 Eggebrecht $\mathrm{H}$, Mehta $\mathrm{RH}$, Metozounve $\mathrm{H}$, et al. Clinical implications of systemic inflammatory response syndrome following thoracic aortic stent-graft placement. J Endovasc Ther 2008;15 (02):135-143

60 Gorla R, Erbel R, Kahlert P, et al. Clinical features and prognostic value of stent-graft-induced post-implantation syndrome after thoracic endovascular aortic repair in patients with type B acute aortic syndromes. Eur J Cardiothorac Surg 2016;49(04): 1239-1247

61 Gorla R, Erbel R, Eagle KA, Bossone E. Systemic inflammatory response syndromes in the era of interventional cardiology. Vascul Pharmacol 2018;107:53-66 
214 Management and Outcomes of Acute Aortic Syndromes: The Mainz-Essen Experience Bossone et al.

62 Grabenwöger M, Alfonso F, Bachet J, et al. Thoracic endovascular aortic repair (TEVAR) for the treatment of aortic diseases: a position statement from the European Association for CardioThoracic Surgery (EACTS) and the European Society of Cardiology (ESC), in collaboration with the European Association of Percuta- neous Cardiovascular Interventions (EAPCI). Eur Heart J 2012;33 (13):1558-1563

63 Tsagakis $\mathrm{K}$, Jakob $\mathrm{H}$. Which frozen elephant trunk offers the optimal solution? Reflections from Essen Group. Semin Thorac Cardiovasc Surg 2019;31(04):679-685 